\title{
A New Impact Time and Angle Control Guidance Law for Stationary and Nonmaneuvering Targets
}

\author{
Zhe Yang, Hui Wang, Defu Lin, and Luyao Zang \\ School of Aerospace Engineering, Beijing Institute of Technology, Beijing 100081, China \\ Correspondence should be addressed to Hui Wang; wh20031131@126.com
}

Received 25 January 2016; Accepted 2 August 2016

Academic Editor: Wen Bao

Copyright (C) 2016 Zhe Yang et al. This is an open access article distributed under the Creative Commons Attribution License, which permits unrestricted use, distribution, and reproduction in any medium, provided the original work is properly cited.

\begin{abstract}
A guidance problem for impact time and angle control applicable to cooperative attack is considered based on the sliding mode control. In order to satisfy the impact angle constraint, a line-of-sight rate polynomial function is introduced with four tuning parameters. And the time-to-go derivative with respect to a downrange orientation is derived to minimize the impact time error. Then the sliding mode control surface with impact time and angle constraints is constructed using nonlinear engagement dynamics to provide an accurate solution. The proposed guidance law is easily extended to a nonmaneuvering target using the predicted interception point. Numerical simulations are performed to verify the effectiveness of the proposed guidance law for different engagement scenarios.
\end{abstract}

\section{Introduction}

In modern warfare, the objectives of guidance laws are not only limited to the interception of targets with zero miss distance. To be effective, guidance laws also account for terminal constraints such as the impact time and impact angle [1]. In some applications, it is desirable to control the heading angle of missiles such as antitank missiles and antiship missiles to increase warhead effectiveness and lethality. The impact time constraint is important when performing salvo attacks to enhance the survivability of the missiles against advanced self-defense systems of battleships, such as close-in weapon systems (CIWS) [2]. Typical warships have two to four CIWS to cover a certain defensive zone [3]. A simultaneous attack of multiple missiles with impact time and angle control is regarded as a cost-effective and efficient cooperative attack strategy.

Guidance laws considering terminal angle constraint have been widely studied in the past decades. One of the initial efforts in this research field was the proposal of a suboptimal guidance law for reentry vehicles with impact angle control by Kim and Grider [4]. Subsequently, Kim et al. [5] derived a biased proportional navigation guidance law which included an additional time-varying bias term to impose a desired attitude angle. A generalized form of the optimal guidance law considering the terminal angle constraint was studied in [6], in which two time-to-go calculation methods were proposed to account for path curvature. In addition to using optimal control theory, sliding mode control was also used to achieve terminal angle control [7-9], which was mostly based on nonsingular terminal sliding mode [7], second-order sliding mode [8], and finite-time convergent sliding mode control [9].

The design of impact time control guidance laws was also an active research area. Jeon et al. [10] first derived a suboptimal guidance law to control the impact time against a stationary target, which combined the proportional navigation guidance command and the feedback of the impact time error. Motivated by the idea of ITCG, Zhao and Zhou [11] designed centralized and distributed coordination algorithms for multimissile salvo attacks. Sliding mode control was also used to design impact time control guidance laws. In [12], the switching surface was defined as a combination of the impact time error and the line-of-sight (LOS) rate to meet the requirement of the impact time and zero miss distance. In [13], another nonsingular sliding mode guidance law was proposed for the impact time problem, in which a positive continuous nonlinear function of the lead angle was 
introduced to avoid the singularity of the guidance command. In addition, the impact time control guidance problems were also extended to three dimensions $[14,15]$.

Though the issues of impact time and angle control have been studied separately, only a few papers consider both constraints simultaneously. Bokyung and Youdan [16] first combined an impact angle guidance law and an impact time control guidance law to enhance high survivability and kill probabilities. Another method of accounting for both constraints was to derive an impact angle biased proportional guidance law with an additional command for the impact time [17]. Harl and Balakrishnan solved this impact time and angle control problem by shaping the LOS rate and tracking it with second-order sliding mode control [18]. The LOS rate shaping profile was established as a fourth-order polynomial with five tuning parameters to satisfy both impact time and angle constraints. However, the impact time control parameters had to be calculated using an offline complex algorithm for each engagement scenario. Recently, a new strategy was presented based on switching between an impact time control guidance law and an impact angle guidance law [19]. In this scheme, the guidance law corrected the impact time in its first step and then switched to an anglecontrol guidance law when the time error was reduced to an acceptable value. The potential drawback of this guidance law is that its acceleration command may jump suddenly at the switching instant. An abrupt command change is unfavorable for the missile because it may cause autopilot instability due to the actuator's slew rate limit [20].

In this paper, a Lyapunov-based impact time and angle control guidance (ITACG) law is proposed. The guidance law is initially developed against a stationary target and then extended to a nonmaneuvering target. The switching surface is defined as a combination of the impact time error and the LOS rate error for satisfying the impact time and angle requirements. The effectiveness of the proposed guidance law is illustrated by numerical simulations under different scenarios such as the many-to-one engagement and the presence of autopilot delay. Simulations highlight the viability and robustness of the guidance law. Compared with other methods in the literatures $[18,19]$, the proposed guidance law does not require the offline algorithm to calculate the impact time parameter and there is no abrupt jump in the acceleration command during the engagement.

The remainder of this paper is organized as follows. Section 2 defines the problem that is considered in this work. Section 3 derives the proposed impact time and angle control guidance law, followed by numerical simulations to evaluate its performance in Section 4. Finally, some conclusions are given in Section 5.

\section{Problem Statement}

To define the problem, we first consider a two-dimensional homing guidance geometry for a stationary target as shown in Figure 1. The initial positions of the missile and the target are denoted as $\left(x_{0}, y_{0}\right)$ and $\left(x_{T}, y_{T}\right) . r, \theta, \lambda, \sigma$ denote range-togo, heading angle, LOS angle, and leading angle, respectively.

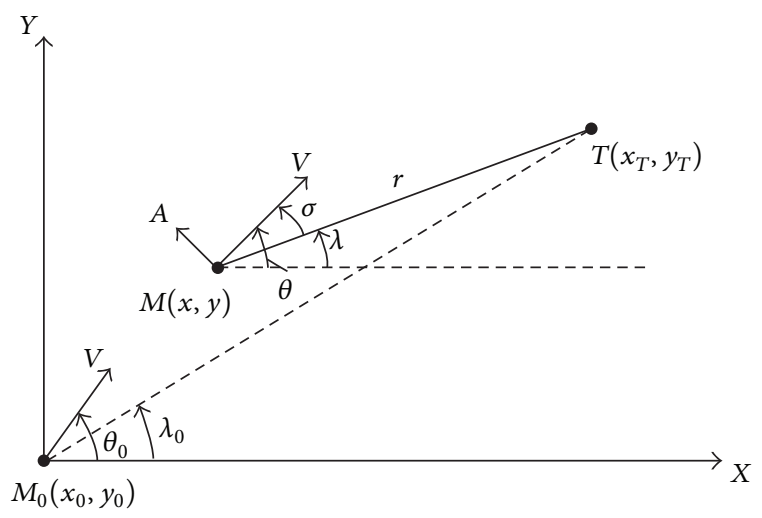

FIGURE 1: Missile/target engagement geometry.

$A$ is the missile acceleration, which is perpendicular to the constant missile velocity $V$.

The equations of motion for the missile are given by

$$
\begin{aligned}
& \frac{d x}{d t}=V \cos \theta, \\
& \frac{d y}{d t}=V \sin \theta, \\
& \frac{d \theta}{d t}=\frac{A}{V}
\end{aligned}
$$

and its boundary conditions are given as

$$
\begin{gathered}
x\left(t_{0}\right)=x_{0}, \\
y\left(t_{0}\right)=x_{0}, \\
\theta\left(t_{0}\right)=\theta_{0}, \\
x\left(t_{f}\right)=x_{f}, \\
y\left(t_{f}\right)=y_{f}, \\
\theta\left(t_{f}\right)=\theta_{d}, \\
t_{f}=T_{d},
\end{gathered}
$$

where the subscripts 0 and $f$ denote the initial and terminal instant. The boundary conditions require the missile to intercept the target at the impact time $T_{d}$ and the impact angle $\theta_{d}$.

The engagement kinematics are expressed from Figure 1 as

$$
\begin{aligned}
& \frac{d \lambda}{d t}=-\frac{V \sin (\theta-\lambda)}{r}, \\
& \frac{d r}{d t}=-V \cos (\theta-\lambda) .
\end{aligned}
$$

For the impact time and angle control problem, it is more convenient to have a set of equations with respect to downrange $x$ as the independent variable instead of time $t$. This is because the final downrange always corresponds to 
the target's position $x_{T}$ while the final time $t_{f}$ varies with the choice of impact time. The conversion between time $t$ and downrange $x$ can be performed by using some variable $\alpha$ as follows [18]:

$$
\frac{d \alpha}{d x}=\frac{d \alpha}{d t} \frac{d t}{d x}
$$

Under this conversion, (1) can be rewritten as

$$
\begin{aligned}
& t^{\prime}=\frac{d t}{d x}=\frac{1}{V \cos \theta}, \\
& y^{\prime}=\frac{d y}{d x}=\tan \theta \\
& \theta^{\prime}=\frac{d \theta}{d x}=\frac{A}{V^{2} \cos \theta}
\end{aligned}
$$

In (5), the "prime" superscript denotes that the new differentiation is performed with respect to downrange $x$. The same conversion process can be also applied to the boundary conditions in (2) and the engagement kinematics in (3), leading to

$$
\begin{aligned}
& y\left(x_{0}\right)=y_{0}, \\
& \theta\left(x_{0}\right)=\theta_{0}, \\
& t\left(x_{0}\right)=0, \\
& y\left(x_{T}\right)=y_{T}, \\
& \theta\left(x_{T}\right)=\theta_{d}, \\
& t\left(x_{T}\right)=T_{d}, \\
& \lambda^{\prime}(x)=-\frac{\sin (\theta-\lambda)}{r \cos \theta}, \\
& r^{\prime}(x)=-\frac{\cos (\theta-\lambda)}{\cos \theta} .
\end{aligned}
$$

\section{Impact Time and Angle Control Guidance}

3.1. Desired LOS Angle and Angular Rate Shaping. In this subsection, motivated by the study in [18], an approach for shaping the LOS rate profile is derived to achieve impact angle control. The desired LOS angle and angular rate can be defined as polynomial functions with respect to downrange $x$, which are expressed as

$$
\begin{aligned}
& \lambda_{d}(x)=a \frac{x^{3}}{x_{T}^{3}}+b \frac{x^{2}}{x_{T}^{2}}+c \frac{x}{x_{T}}+d, \\
& \lambda_{d}^{\prime}(x)=3 a \frac{x^{2}}{x_{T}^{3}}+2 b \frac{x}{x_{T}^{2}}+c \frac{1}{x_{T}},
\end{aligned}
$$

where $\lambda_{d}(x)$ and $\lambda_{d}^{\prime}(x)$ are the desired LOS angle and LOS rate, respectively. The symbols $a, b, c$, and $d$ represent four constant coefficients, which can be solved by using boundary conditions.
The first two boundary conditions are specified by the initial conditions as

$$
\begin{aligned}
& \lambda_{d}\left(x_{0}\right)=\lambda_{0}=\tan ^{-1}\left(\frac{y_{T}-y_{0}}{x_{T}-x_{0}}\right), \\
& \lambda_{d}^{\prime}\left(x_{0}\right)=\lambda_{0}^{\prime}=-\frac{\sin \left(\theta_{0}-\lambda_{0}\right)}{\cos \theta_{0} \sqrt{\left(x_{0}-x_{T}\right)^{2}+\left(y_{0}-y_{T}\right)^{2}}} .
\end{aligned}
$$

To guarantee a hit, the desired LOS rate should be zero at the downrange corresponding to the target location. So the third boundary condition can be expressed as

$$
\lambda_{d}^{\prime}\left(x_{T}\right)=0
$$

When the target is stationary or its speed is far less than the missile velocity, the final heading angle will be equal to the LOS angle since the desired final LOS rate is zero. Thus, another terminal boundary condition that considers the impact angle control is taken as

$$
\lambda_{d}\left(x_{T}\right)=\theta_{d}
$$

where $\theta_{d}$ is the desired terminal angle.

By applying (9), (10), and (11), we can obtain

$$
\begin{aligned}
a \frac{x_{0}^{3}}{x_{T}^{3}}+b \frac{x_{0}^{2}}{x_{T}^{2}}+c \frac{x_{0}}{x_{T}}+d & =\lambda_{0}, \\
3 a \frac{x_{0}^{2}}{x_{T}^{3}}+2 b \frac{x_{0}}{x_{T}^{2}}+c \frac{1}{x_{T}} & =\lambda_{0}^{\prime}, \\
\frac{3 a}{x_{T}}+\frac{2 b}{x_{T}}+\frac{c}{x_{T}} & =0, \\
a+b+c+d & =\theta_{d} .
\end{aligned}
$$

From (12), the four tuning parameters can be calculated as

$$
\begin{aligned}
a= & \frac{x_{T}^{3}}{\left(x_{T}-x_{0}\right)^{2}} \lambda_{0}^{\prime}-\frac{2 x_{T}^{3}\left(\theta_{d}-\lambda_{0}\right)}{\left(x_{T}-x_{0}\right)^{3}}, \\
b= & \frac{3 x_{T}^{2}\left(x_{0}+x_{T}\right)\left(\theta_{d}-\lambda_{0}\right)}{\left(x_{T}-x_{0}\right)^{3}}-\frac{x_{T}^{2}\left(x_{0}+2 x_{T}\right)}{\left(x_{T}-x_{0}\right)^{2}} \lambda_{0}^{\prime}, \\
c= & -\frac{6 x_{0} x_{T}^{2}\left(\theta_{d}-\lambda_{0}\right)}{\left(x_{T}-x_{0}\right)^{3}}+\frac{x_{T}^{2}\left(2 x_{0}+x_{T}\right)}{\left(x_{T}-x_{0}\right)^{2}} \lambda_{0}^{\prime}, \\
d= & \frac{\theta_{d} x_{0}^{2}\left(3 x_{T}-x_{0}\right)+\lambda_{0} x_{T}^{2}\left(x_{T}-3 x_{0}\right)}{\left(x_{T}-x_{0}\right)^{3}} \\
& -\frac{x_{0} x_{T}^{2}}{\left(x_{T}-x_{0}\right)^{2}} \lambda_{0}^{\prime} .
\end{aligned}
$$

The parameters in (13), which are a function of the impact angle $\theta_{d}$, ensure that the missile intercepts the target at the desired terminal angle. 
3.2. Estimation of Time-to-Go. Accurate estimation of timeto-go is vital to control the impact time. A poor estimation of time-to-go not only severely degrades guidance performance but also makes the missile off the desired trajectory. If the missile flies straight along LOS, that is, $\theta=\lambda$, then the timeto-go estimation should be $r / V$. When $\theta-\lambda$ is not zero, but small, the time-to-go estimation can be approximated as [21]

$$
t_{\mathrm{go}}=\frac{r}{V}\left(1+\frac{\sigma^{2}}{2(2 N-1)}\right),
$$

where $N$ denotes the effective navigation constant.

For the impact time and angle control, the time-to-go estimation in (14) may not be adequate because its time-togo error only depends on the initial heading error. But the effect of the terminal angle constraint is not considered. In [6], the trajectory considering the impact angle constraint can be approximated by a third-order polynomial of range-to-go. Thus the time-to-go estimation can be calculated as the range over the average velocity, which gives

$$
t_{\mathrm{go}} \approx \frac{r}{V}\left(1+\frac{\sigma^{2}+\sigma_{f}^{2}}{15}-\frac{\sigma \sigma_{f}}{30}\right),
$$

where $\sigma$ and $\sigma_{f}$ are defined as

$$
\begin{gathered}
\sigma=\theta-\lambda, \\
\sigma_{f}=\theta_{d}-\lambda .
\end{gathered}
$$

By differentiating (15), the derivative of time-to-go $t_{\text {go }}^{\prime}$ with respect to downrange $x$ can be calculated as

$$
\begin{aligned}
t_{\mathrm{go}}^{\prime}= & \frac{r^{\prime}}{V}\left(1+\frac{\sigma^{2}+\sigma_{f}^{2}}{15}-\frac{\sigma \sigma_{f}}{30}\right) \\
& +\frac{r}{V}\left(\frac{2 \sigma \sigma^{\prime}+2 \sigma_{f} \sigma_{f}^{\prime}}{15}-\frac{\sigma^{\prime} \sigma_{f}+\sigma \sigma_{f}^{\prime}}{30}\right) .
\end{aligned}
$$

By differentiating (16), we can also obtain the derivatives of $\sigma$ and $\sigma_{f}$ as

$$
\begin{aligned}
& \sigma^{\prime}=\theta^{\prime}-\lambda^{\prime}=\frac{A}{V^{2} \cos \theta}-\lambda^{\prime}, \\
& \sigma_{f}^{\prime}=-\lambda^{\prime} .
\end{aligned}
$$

Substituting (18) into (17) yields

$$
\begin{aligned}
t_{\mathrm{go}}^{\prime}= & \frac{r^{\prime}}{V}\left(1+\frac{\sigma^{2}+\sigma_{f}^{2}}{15}-\frac{\sigma \sigma_{f}}{30}\right) \\
& +\frac{r A}{V^{3} \cos \theta}\left(\frac{4 \sigma-\sigma_{f}}{30}\right)-\frac{r \lambda^{\prime}\left(\sigma+\sigma_{f}\right)}{10 V} .
\end{aligned}
$$

Equation (19) can be rewritten in a simpler form as

$$
t_{\mathrm{go}}^{\prime}=K_{1}+K_{2} A
$$

where

$$
\begin{aligned}
& K_{1}=\frac{r^{\prime}}{V^{2}}\left(1+\frac{\sigma^{2}+\sigma_{f}^{2}}{15}-\frac{\sigma \sigma_{f}}{30}\right)-\frac{r \lambda^{\prime}}{10 V}\left(\sigma+\sigma_{f}\right), \\
& K_{2}=\frac{r}{V^{3} \cos \theta}\left(\frac{2 \sigma}{15}-\frac{\sigma_{f}}{30}\right) .
\end{aligned}
$$

3.3. Impact Time and Angle Control Guidance Law. The objective of the guidance law is to enable the missile to intercept a target with the desired impact time and impact angle. According to the above requirements, the switching surface with respect to downrange is chosen as

$$
s=e_{\lambda^{\prime}}+e_{t} \operatorname{sign}\left(e_{\lambda^{\prime}}\right)
$$

where

$$
\begin{aligned}
e_{\lambda^{\prime}} & =\lambda_{d}^{\prime}(x)-\lambda^{\prime}(x), \\
\left(t_{\text {elap }}+t_{\text {go }}\right) e_{t} & =T_{d}-\left(t_{\text {elap }}+t_{\text {go }}\right) .
\end{aligned}
$$

In (23), $\lambda^{\prime}$ is the real-time LOS rate and $\lambda_{d}^{\prime}$ is the desired shaping LOS rate in (7). In (23), $t_{\text {elap }}$ is the time elapsed after the launch of the missile. Note that the desired flight time $T_{d}$ should be larger than the initial time-to-go $t_{\text {go }}\left(x_{0}\right)$, and then $e_{t} \geq 0$.

Proposition 1. The sliding surface in (22) can help to achieve both impact time and angle constraints.

Proof. The dynamics of the sliding surface in (22) are given by $s=0$; that is,

$$
\begin{aligned}
e_{\lambda^{\prime}}+e_{t} \operatorname{sign}\left(e_{\lambda^{\prime}}\right) & =0 \Longrightarrow \\
e_{\lambda^{\prime}} & =-e_{t} \operatorname{sign}\left(e_{\lambda^{\prime}}\right) .
\end{aligned}
$$

When the LOS rate error $e_{\lambda^{\prime}}$ is not equal to zero, we can get

$$
\left|e_{\lambda^{\prime}}\right|=-e_{t} \leq 0
$$

But $\left|e_{\lambda^{\prime}}\right| \geq 0$ for all $e_{\lambda^{\prime}}$, so the only term is $e_{t}=0, e_{\lambda^{\prime}}=0$. In other words, the sliding mode $s=0$ cannot occur until $e_{t}=0$ and $e_{\lambda^{\prime}}=0 . e_{t}=0$ means that the estimated interception time is equal to the desired impact time. $e_{\lambda^{\prime}}=0$ means that the real-time LOS rate is equal to the designed one in (7) at the interception point; that is, $\lambda^{\prime}\left(x_{T}\right)=\lambda_{d}^{\prime}\left(x_{T}\right)$. According to the terminal boundary conditions in (10) and (11), we can get $\lambda^{\prime}\left(x_{T}\right)=\lambda_{d}^{\prime}\left(x_{T}\right)=0$ and $\lambda\left(x_{T}\right)=\lambda_{d}\left(x_{T}\right)=\theta_{d}$. Thus the angle constraint can be achieved.

Now consider the case $e_{t} \neq 0$ and $e_{\lambda^{\prime}}=0$ at the start of the engagement. Differentiating (23) yields

$$
\begin{aligned}
e_{\lambda^{\prime}}^{\prime}= & \lambda_{d}^{\prime \prime}(x)-\lambda^{\prime \prime}(x) \\
= & \frac{\cos (\theta-\lambda)+\tan \theta \sin (\theta-\lambda)}{r V^{2} \cos ^{2} \theta} A \\
& +\frac{\sin (2 \theta-2 \lambda)}{r^{2} \cos ^{2} \theta}+\frac{6 a x}{x_{T}^{3}}+\frac{2 b}{x_{T}^{2}} \\
= & \frac{\cos \lambda}{r V^{2} \cos ^{3} \theta} A+\frac{\sin (2 \theta-2 \lambda)}{r^{2} \cos ^{2} \theta}+\frac{6 a x}{x_{T}^{3}}+\frac{2 b}{x_{T}^{2}} .
\end{aligned}
$$


Note that $e_{\lambda^{\prime}}^{\prime}$ is not identically equal to zero with the change of range-to-go $r$, lateral acceleration $A$, heading angle $\theta$, and LOS angle $\lambda$. Hence, $e_{\lambda^{\prime}}$ is a time-varying value and $e_{\lambda^{\prime}}=0$ is not an attractor while $s \neq 0$. Once $e_{\lambda^{\prime}}$ is not equal to zero, we can use the sliding surface above to control $e_{t}$ and $e_{\lambda^{\prime}}$.

To design lateral acceleration $A$ for impact time and angle control, let us consider the downrange derivative of the switching surface.

Case 1. When LOS rate is less than the desired LOS rate, that is, $e_{\lambda^{\prime}}>0$, then from (22)

$$
s=e_{\lambda^{\prime}}+e_{t} .
$$

Differentiating (28) yields

$$
\begin{aligned}
s^{\prime}(x)= & e_{\lambda^{\prime}}^{\prime}(x)+e_{t}^{\prime}(x) \\
= & \lambda_{d}^{\prime \prime}(x)-\lambda^{\prime \prime}(x)-\left(t_{\text {elap }}^{\prime}+t_{\mathrm{go}}^{\prime}\right) \\
= & \frac{\cos \lambda}{r V^{2} \cos ^{3} \theta} A+\frac{2 r^{\prime} \lambda^{\prime}}{r}+\frac{6 a x}{x_{T}^{3}}+\frac{2 b}{x_{T}^{2}}-\frac{1}{V \cos \theta} \\
& -\left(K_{1}+K_{2} A\right) .
\end{aligned}
$$

Based on (29), one can design the lateral acceleration using the Lyapunov-based nonlinear control theory as the sum of an equivalent control and a discontinuous control. Consider

$$
A=a_{M}^{\mathrm{eq}}+a_{M}^{\mathrm{dis}} \text {. }
$$

The equivalent component $a_{M}^{\mathrm{eq}}$ is obtained by setting $s^{\prime}=0$, which gives

$$
a_{M}^{\mathrm{eq}}=\frac{1 / V \cos \theta+K_{1}-\left(2 r^{\prime} \lambda^{\prime} / r+6 a x / x_{T}^{3}+2 b / x_{T}^{2}\right)}{\cos \lambda / r V^{2} \cos ^{3} \theta-K_{2}} .
$$

Once the system reaches the sliding mode, the equivalent control component keeps the system on the sliding mode. In addition, the discontinuous component guarantees that the switching surface $s$ converges to zero, which is designed in the form of

$$
a_{M}^{\mathrm{dis}}=\frac{-M \operatorname{sign}(s)}{\operatorname{sign}\left(\cos \lambda / r V^{2} \cos ^{3} \theta-K_{2}\right)},
$$

where $M$ denotes the control magnitude, $M>0$. By increasing the control magnitude $M$, the time interval to reach sliding mode is reduced. However, $M$ should be chosen appropriately to ensure that the acceleration constraint is not violated.

Consider the Lyapunov function candidate to be $V_{L}=$ $(1 / 2) s^{2}$. Differentiating $V_{L}$ and substituting (29) and (30) into the resulting expression gives

$$
V_{L}^{\prime}=s s^{\prime}=-M|s|\left|\frac{\cos \lambda}{r V^{2} \cos ^{3} \theta}-K_{2}\right|,
$$

which is negative definite if

$$
\frac{\cos \lambda}{r V^{2} \cos ^{3} \theta}-K_{2} \neq 0
$$

Under these conditions, it is guaranteed to achieve the sliding mode. Hence the guidance law is

$$
\begin{aligned}
A= & a_{M}^{\mathrm{eq}}+a_{M}^{\mathrm{dis}} \\
= & \frac{1 / V \cos \theta+K_{1}-\left(2 r^{\prime} \lambda^{\prime} / r+6 a x / x_{T}^{3}+2 b / x_{T}^{2}\right)}{\cos \lambda / r V^{2} \cos ^{3} \theta-K_{2}} \\
& +\frac{-M \operatorname{sign}(s)}{\operatorname{sign}\left(\cos \lambda / r V^{2} \cos ^{3} \theta-K_{2}\right)} .
\end{aligned}
$$

Note that if (34) is not satisfied, then $V_{L}^{\prime}=0$, and the Lyapunov condition will not be satisfied. If (34) is violated, then

$$
\frac{\cos \lambda}{r V^{2} \cos ^{3} \theta}-K_{2}=0
$$

After substituting (21) and neglecting smaller terms of $K_{2}$, we can get

$$
\frac{15 V}{2 r^{2}}=\frac{\cos ^{2} \theta}{\cos \lambda} \sigma
$$

The left hand side (LHS) of (37) remains positive during the whole engagement. Here we consider the range of $\theta$ and $\lambda$ as $|\theta|<\pi / 2,|\lambda|<\pi / 2$. If the heading angle error $\sigma \leq 0$ then $\sigma \cos ^{2} \theta / \cos \lambda \leq 0$, and the condition (34) is not violated; If $\sigma>0$, that is, $\theta>\lambda$, the right hand side is greater than zero. According to the terminal boundary conditions in (10) and (11), that is, $\lambda^{\prime}\left(x_{T}\right)=0$ and $\lambda\left(x_{T}\right)=\theta_{d}$, the heading angle error $\sigma$ converges to zero asymptotically. The highly increasing time-varying term $1 / r^{2}$ on LHS forces the system cross (37) and therefore Lyapunov stability criteria is still satisfied.

Case 2. When LOS rate is greater than the desired LOS rate, that is, $e_{\lambda^{\prime}}<0$, then from (22)

$$
s=e_{\lambda^{\prime}}-e_{t}
$$

Differentiating (38) yields

$$
\begin{aligned}
s^{\prime}= & e_{\lambda^{\prime}}^{\prime}-e_{t}^{\prime}=\lambda_{d}^{\prime \prime}(x)-\lambda^{\prime \prime}(x)+\left(t_{\text {elap }}^{\prime}+t_{\text {go }}^{\prime}\right) \\
= & \frac{\cos \lambda}{r V^{2} \cos ^{3} \theta} A+\frac{2 r^{\prime} \lambda^{\prime}}{r}+\frac{6 a x}{x_{T}^{3}}+\frac{2 b}{x_{T}^{2}}+\frac{1}{V \cos \theta} \\
& +\left(K_{1}+K_{2} A\right) .
\end{aligned}
$$

Also, the lateral acceleration of the missile is designed to be the sum of an equivalent control and a discontinuous control. Consider

$$
A=a_{M}^{\mathrm{eq}}+a_{M}^{\mathrm{dis}}
$$

By setting $s^{\prime}=0$, the equivalent component $a_{M}^{\mathrm{eq}}$ is obtained as

$$
a_{M}^{\mathrm{eq}}=-\frac{1 / V \cos \theta+K_{1}+2 r^{\prime} \lambda^{\prime} / r+6 a x / x_{T}^{3}+2 b / x_{T}^{2}}{\cos \lambda / r V^{2} \cos ^{3} \theta+K_{2}} .
$$


The discontinuous controller, which drives the system towards the switching surface, is of the form

$$
a_{M}^{\mathrm{dis}}=\frac{-M \operatorname{sign}(s)}{\operatorname{sign}\left(\cos \lambda / r V^{2} \cos ^{3} \theta+K_{2}\right)},
$$

where $M$ denotes the control magnitude, $M>0$. Now we consider the Lyapunov function candidate to be $V_{L}=(1 / 2) s^{2}$. Differentiating $V_{L}$ and substituting (39) and (40) into the resulting expression gives

$$
V_{L}^{\prime}=s s^{\prime}=-M|s|\left|\frac{\cos \lambda}{r V^{2} \cos ^{3} \theta}+K_{2}\right| \leq 0,
$$

which is negative definite if

$$
\frac{\cos \lambda}{r V^{2} \cos ^{3} \theta}+K_{2} \neq 0
$$

Under these conditions, it is guaranteed to achieve the sliding mode. Hence the guidance law is

$$
\begin{aligned}
A= & a_{M}^{\mathrm{eq}}+a_{M}^{\mathrm{dis}} \\
= & -\frac{1 / V \cos \theta+K_{1}+2 r^{\prime} \lambda^{\prime} / r+6 a x / x_{T}^{3}+2 b / x_{T}^{2}}{\cos \lambda / r V^{2} \cos ^{3} \theta+K_{2}} \\
& +\frac{-M \operatorname{sign}(s)}{\operatorname{sign}\left(\cos \lambda / r V^{2} \cos ^{3} \theta+K_{2}\right)} .
\end{aligned}
$$

Note that if (44) is not satisfied, $V_{L}^{\prime}=0$, and the Lyapunov condition will not be satisfied. Similarly to the analytical method in Case 1, it can be easily shown that condition (44) is satisfied most of the time during the engagement and hence the impact time and angle criterion can be achieved.

In addition, to reduce chattering due to the application of the discontinuous control in (35) and (45), the discontinuous function $\operatorname{sign}(s)$ can be approximated by the sigmoid function [19]. Consider

$$
\operatorname{sgmf}(s)=2\left(\frac{1}{1+\exp (-s / \varepsilon)}-\frac{1}{2}\right),
$$

where $\varepsilon$ denotes the width of the boundary layer in the sigmoid function. In (46), the function uses a continuous approximation of the discontinuous control when $|s| \leq \varepsilon$. The performance of the sigmoid function as $\varepsilon$ is varied is shown in Figure 2. It can be seen that sigmoid function comes closer to the actual signum function for lower values of $\varepsilon$. This method ensures that the proposed guidance law generates a continuous and smooth lateral acceleration.

3.4. Extension to Nonmaneuvering Targets. The proposed guidance law can be extended to attack a nonmaneuvering target using the concept of predicted interception point (PIP) represented in [22]. The PIP is defined as a virtual point where the missile is expected to intercept the target. The target is assumed to have a speed $V_{T}$ with a heading angle $\theta_{T}$, and the virtual position $\left(x_{T P} y_{T P}\right)$ can be calculated online as follows:

$$
\begin{aligned}
& x_{T P}=x_{T}+V_{T} \cos \left(\theta_{T}\right) t_{\mathrm{go}}, \\
& y_{T P}=y_{T}+V_{T} \sin \left(\theta_{T}\right) t_{\mathrm{go}},
\end{aligned}
$$

TABLE 1: Simulation parameters for stationary target.

\begin{tabular}{lccccc}
\hline$\left(x_{0}, y_{0}\right) / \mathrm{m}$ & $\left(x_{T}, y_{T}\right) / \mathrm{m}$ & $V /(\mathrm{m} / \mathrm{s})$ & $\theta_{0} / \mathrm{deg}$ & $\theta_{f} / \mathrm{deg}$ & $T_{d} / \mathrm{s}$ \\
\hline$(0,500)$ & $(10000,0)$ & 250 & 30 & -60 & 50 \\
\hline
\end{tabular}

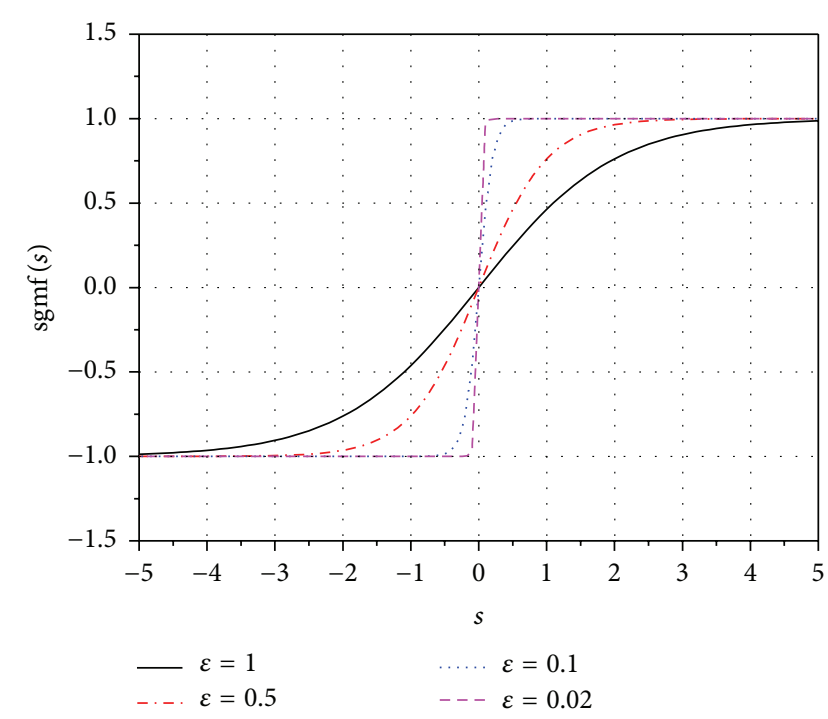

Figure 2: Performance of the sigmoid function.

where $\left(x_{T P}, y_{T P}\right)$ and $\left(x_{T}, y_{T}\right)$ are the predicted interception point and the current position of the target, respectively, and $t_{\mathrm{go}}$ is the time-to-go estimation defined in (15). Using this method, we can approximate a nonmaneuvering target as a virtual stationary target. This concept can easily be incorporated into the proposed ITACG law by replacing $\left(x_{T}, y_{T}\right)$ with $\left(x_{T P}, y_{T P}\right)$.

\section{Simulation Results}

This section presents simulation results of the proposed ITACG law under various engagement scenarios. The proposed guidance law is first simulated in one-to-one and many-to-one scenarios for stationary targets and nonmaneuvering targets. The simulation is then extended to evaluate performance with autopilot lag. For the given engagements, the gain $M$ of the discontinuous component in the proposed guidance law is chosen as $M=50$. The width of the boundary layer in the sigmoid function (46) is chosen as $\varepsilon=0.2$. For each simulation, it is assumed that the desired guidance time and terminal angle are specified.

4.1. Stationary Target. In this subsection, performance of the proposed guidance law considering a stationary target is given. The simulation conditions of the missile are listed in Table 1. In the first simulation, the results of the PNG and sliding mode control guidance (SMCG) [19] law are also shown for comparison with the proposed ITACG. The SMCG requires values for the tolerance parameters $\varepsilon_{1}$ and $\varepsilon_{2}$ in its guidance law switching logic. Once the impact time error becomes smaller than the tolerance parameters, SMCG switches to the impact angle control guidance law. 


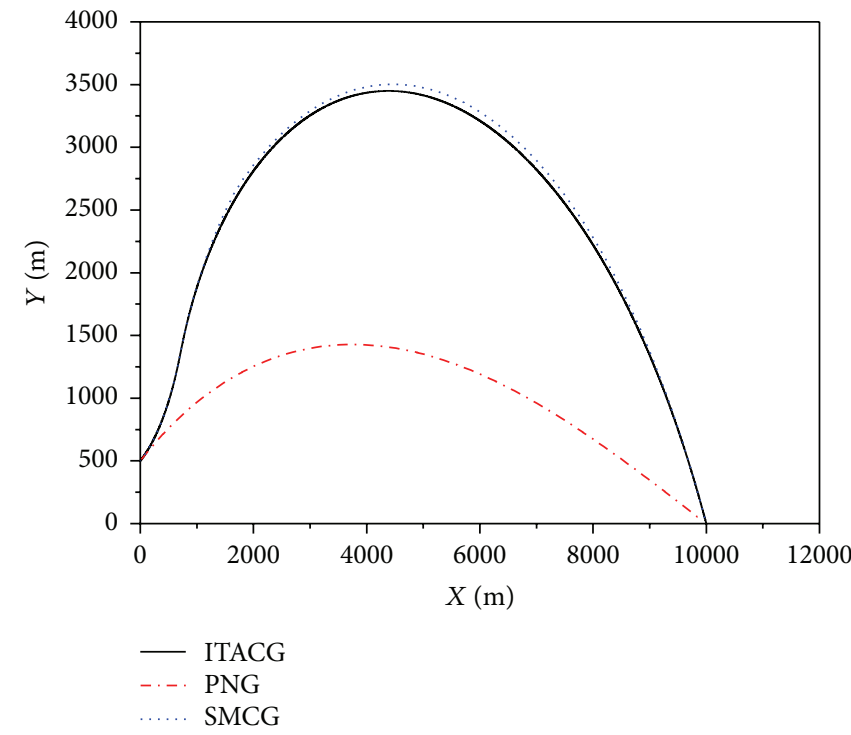

(a) Missile trajectory

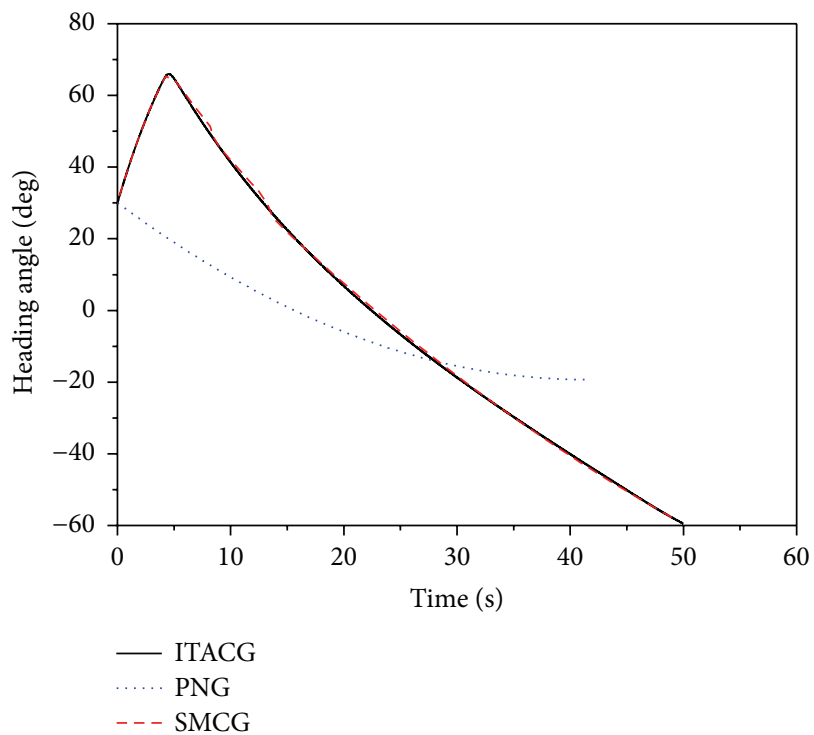

(c) Heading angle
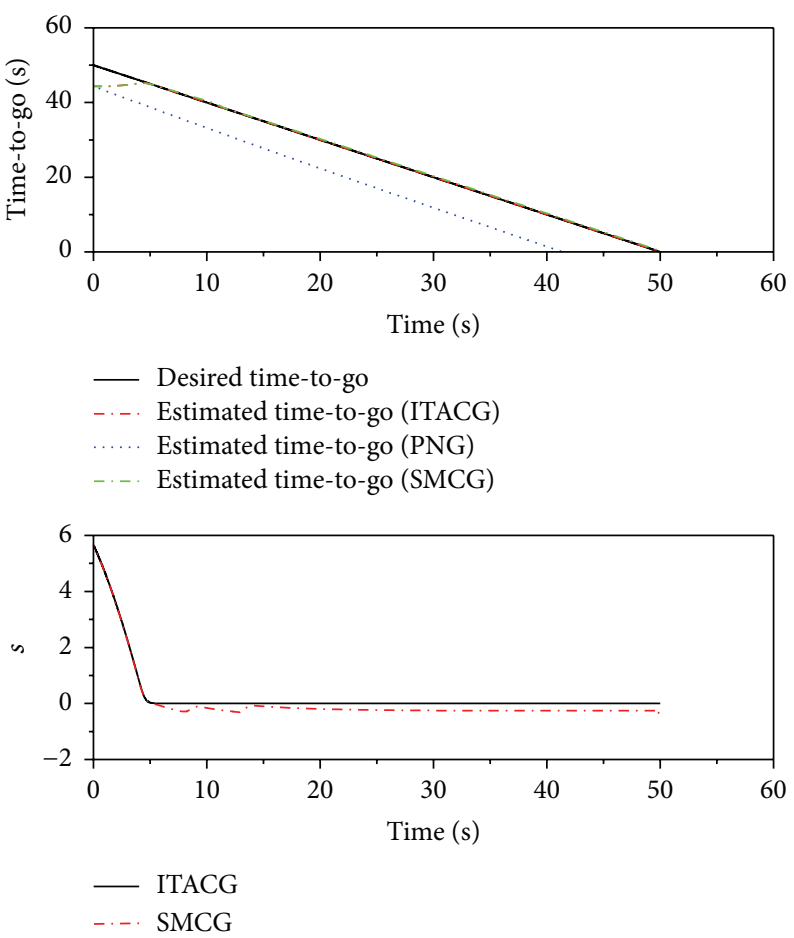

(b) Time-to-go and occurrence of sliding mode

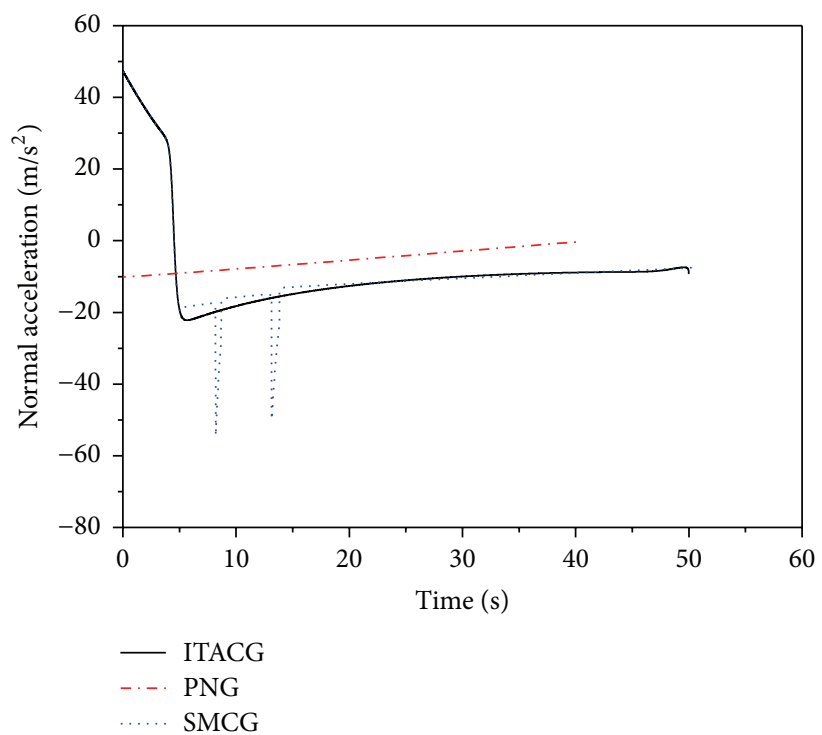

(d) Missile acceleration

FIGURE 3: Simulation results compared with other guidance laws.

Here the tolerance parameters are chosen to be $\varepsilon_{1}=0.3 \mathrm{~s}$ and $\varepsilon_{2}=0.05 \mathrm{~s}$.

The simulation results for this scenario are presented in Figure 3. Figure 3(a) shows that the missile trajectory using ITACG is similar to that of SMCG. Both of these guidance laws use a longer route to achieve the desired impact time $T_{d}$ compared to PNG, which uses a more direct route but hits the target too early. The terminal time of PNG and SMCG in Figure 3(b) can be observed to be $41.41 \mathrm{~s}$ and $50.29 \mathrm{~s}$, respectively. Even though the initial time-to-go of ITACG is about $44.34 \mathrm{~s}$, its final impact time is $49.99 \mathrm{~s}$, which is very close to the desired impact time. Figure 3(b) shows that the sliding surface of ITACG converges to zero quickly, while sliding surface of SMCG is minimized, but never reaches zero. This is because when SMCG switches to the impact angle control guidance law (in other words, the impact time error decreases below the tolerance parameters), it ceases to control the impact time. Figure 3(c) compares the heading angles 


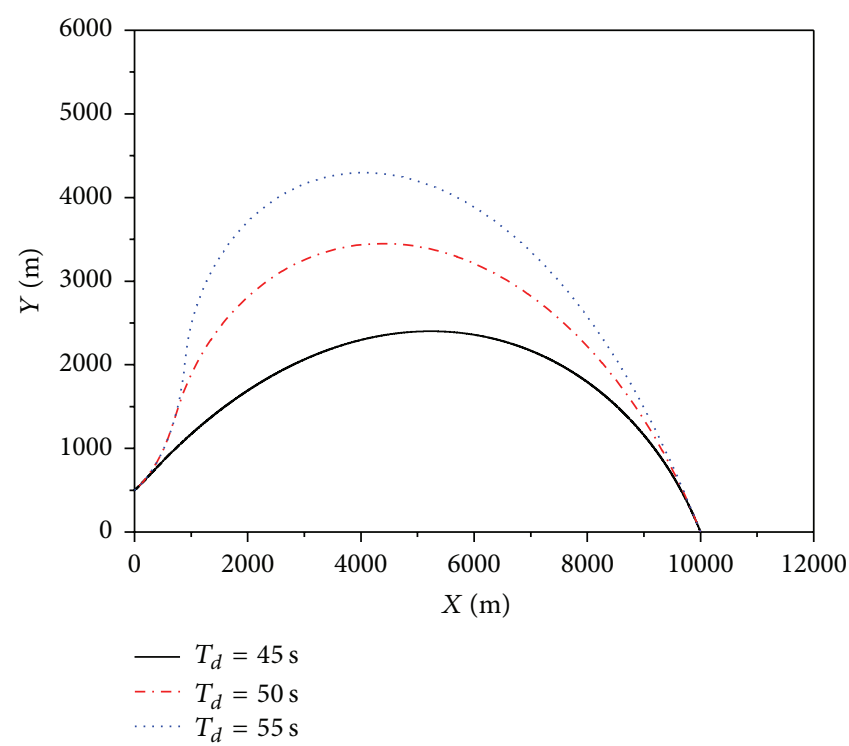

(a) Missile trajectory

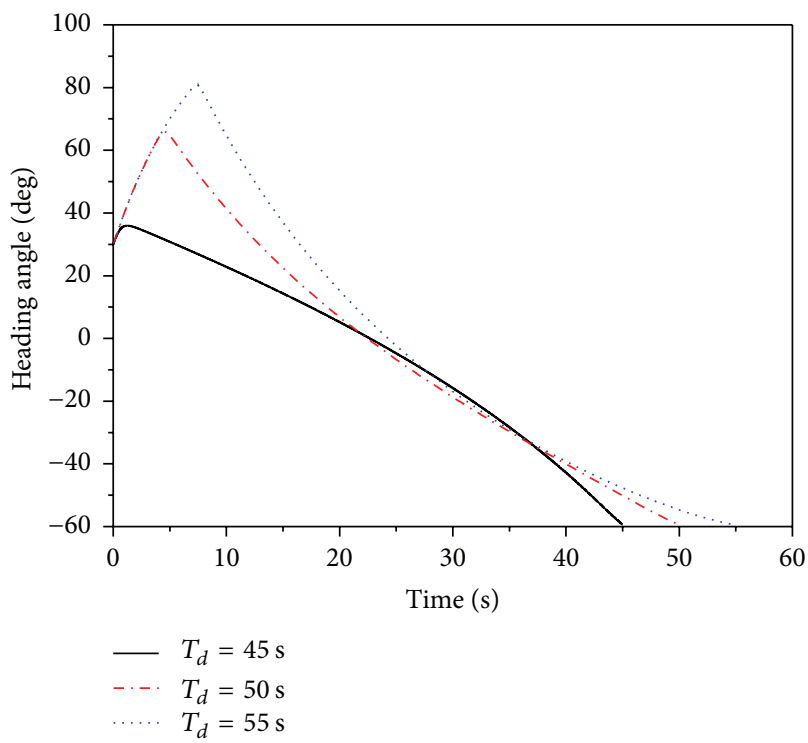

(c) Heading angle
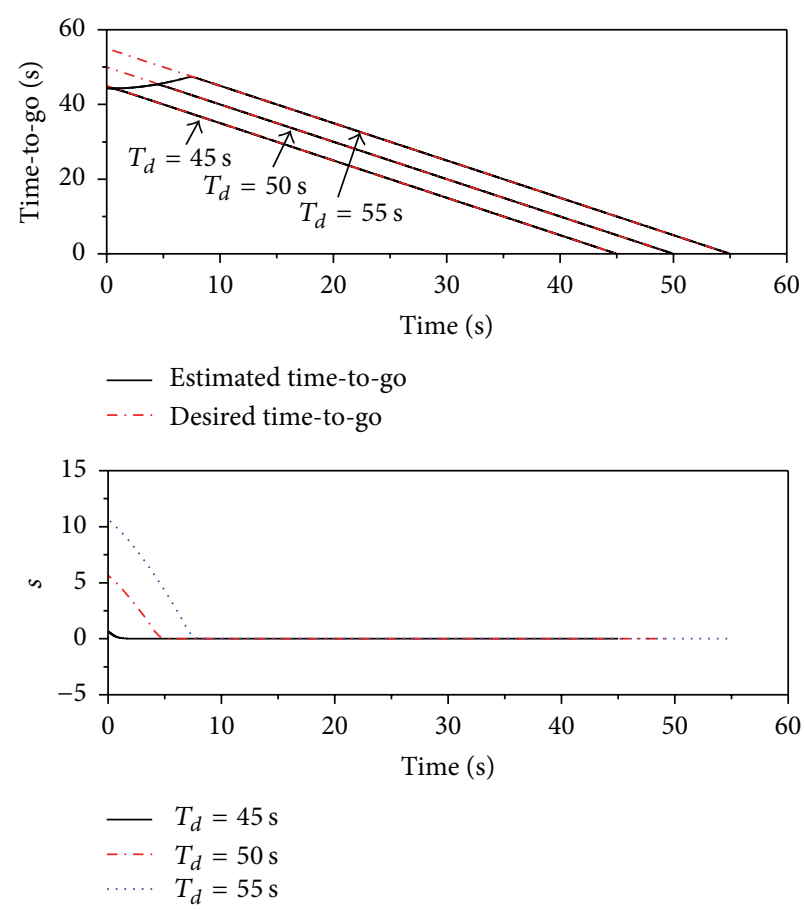

(b) Time-to-go and occurrence of sliding mode

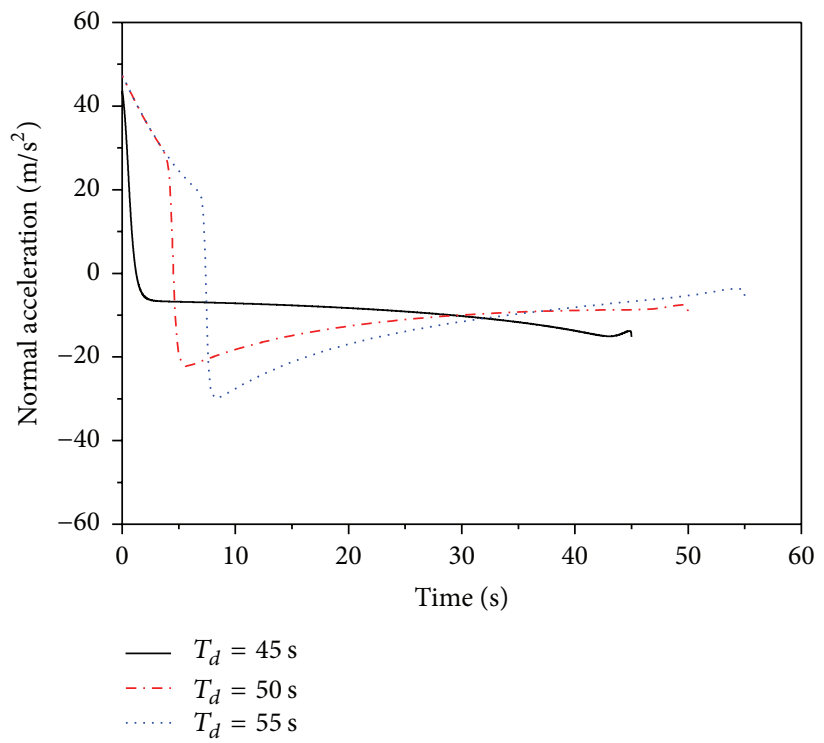

(d) Missile acceleration

FIGURE 4: Simulation results considering various impact times with a fixed impact angle.

of the three guidance laws. The heading angle for ITACG and SMCG increases at the beginning of the engagement, while it decreases monotonically for PNG. However, PNG does not achieve the desired impact angle at the finial time, while ITACG does achieve this goal. It also can be observed from Figure 3(d) that ITACG and SMCG employ higher accelerations than PNG because of the impact time and angle control. Due to the way the guidance logic is implemented in SMCG, its lateral acceleration jumps suddenly at the switching instants. In contrast, the lateral acceleration of
ITACG is observed to change continuously throughout the engagement.

In order to further evaluate the performance of the proposed guidance law, simulations with the impact time of $T_{d}=45,50,55 \mathrm{~s}$ and a fixed impact angle of $\theta_{d}=-60 \mathrm{deg}$ are performed. All other simulation conditions are the same as in the previous scenario in Table 1. Figures 4(a) and 4(b) show that the proposed guidance law generates feasible trajectories for satisfying the three impact time constraints. In order to increase the impact time, the missile needs to turn farther 

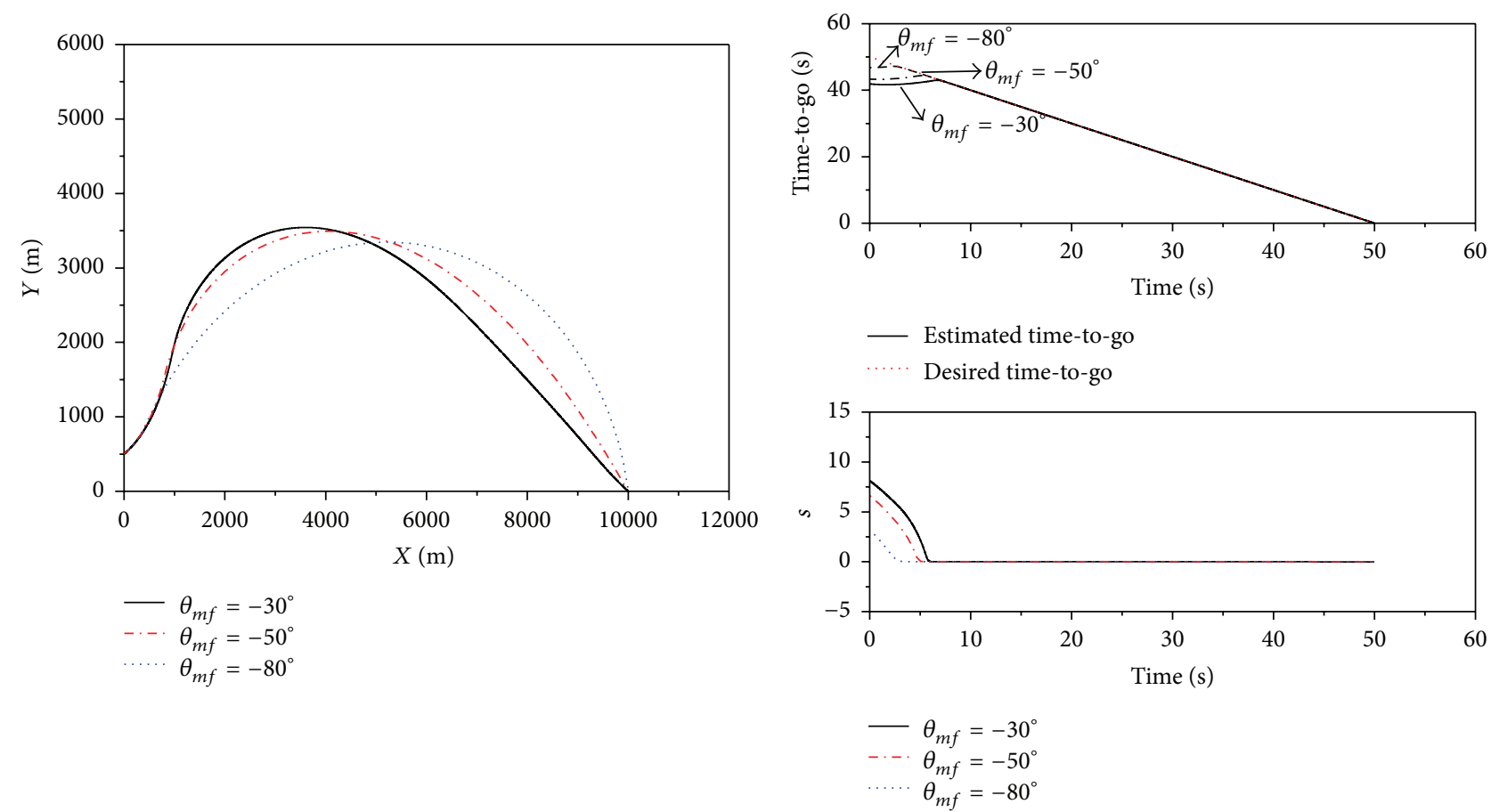

(a) Missile trajectory

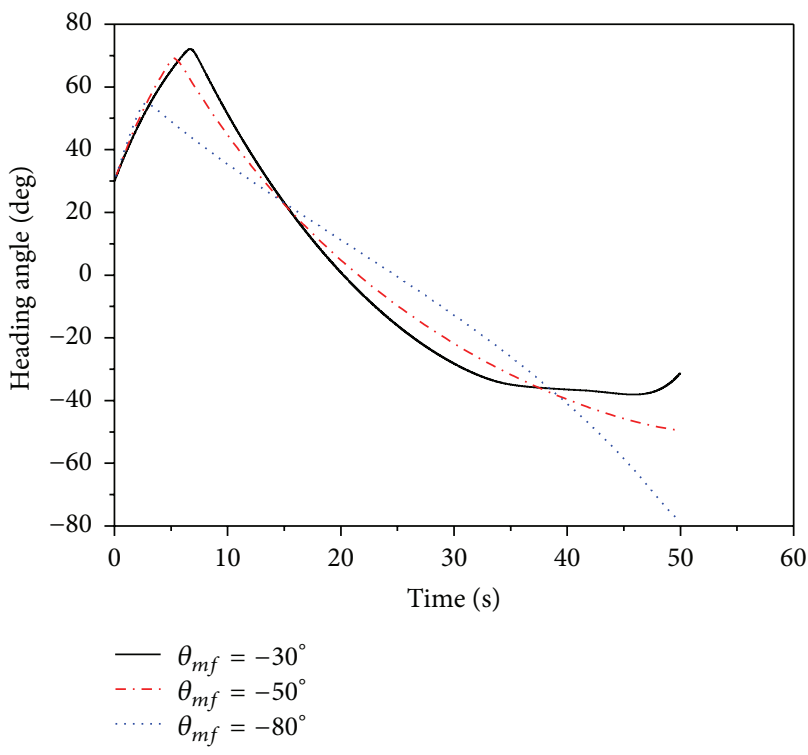

(c) Heading angle (b) Time-to-go and occurrence of sliding mode

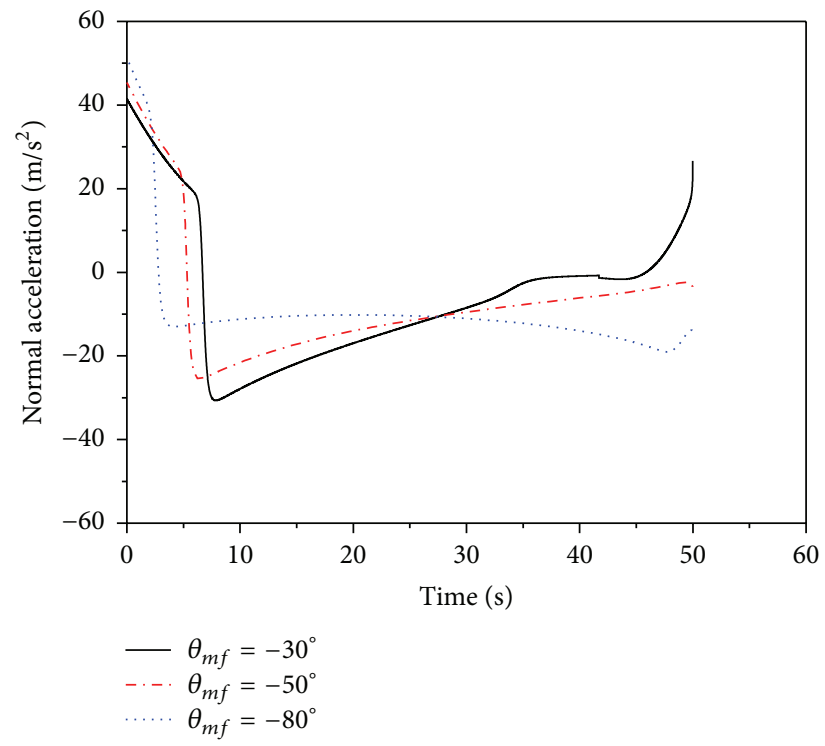

(d) Missile acceleration

FIGURE 5: Simulation results considering various impact angles with a fixed impact time.

away from the target by increasing its acceleration, as shown in Figures 4(c) and 4(d).

Next simulations are conducted with a fixed impact time of $50 \mathrm{~s}$ and desired impact angles of $-30 \mathrm{deg},-50 \mathrm{deg}$, and $-80 \mathrm{deg}$. All other simulation conditions are the same as in the previous scenario in Table 1. Figures 5(a) and 5(c) show that three different trajectories satisfy each impact angle requirement. Figure 5(b) shows that each time-to-go estimation approaches the same desired time-to-go quickly. Figure 5(d) shows that the different angle constraints require the different lateral accelerations. For each case, the acceleration is large at the beginning of the engagement until the system reaches the sliding mode surface.

In the next simulation, a salvo attack engagement scenario is considered with three missiles. Three missiles are assumed to be launched from different positions with different initial heading angles and different impact angles. All three missiles are required to hit the target simultaneously. In this case, each missile has the same speed of $250 \mathrm{~m} / \mathrm{s}$, and the target position is still $(10000,0) \mathrm{m}$. Other relevant 


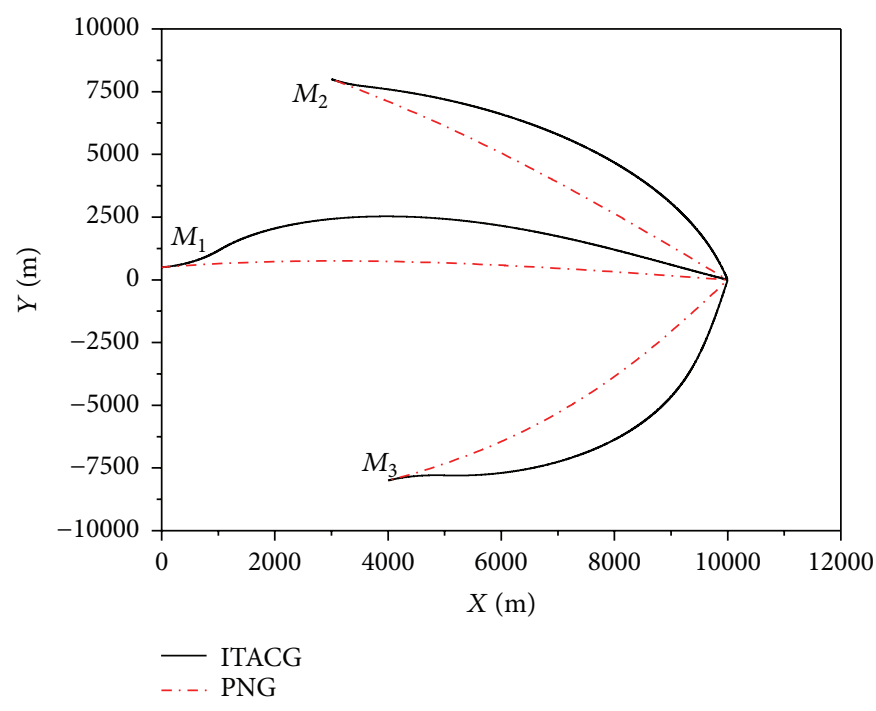

(a) Missile trajectory

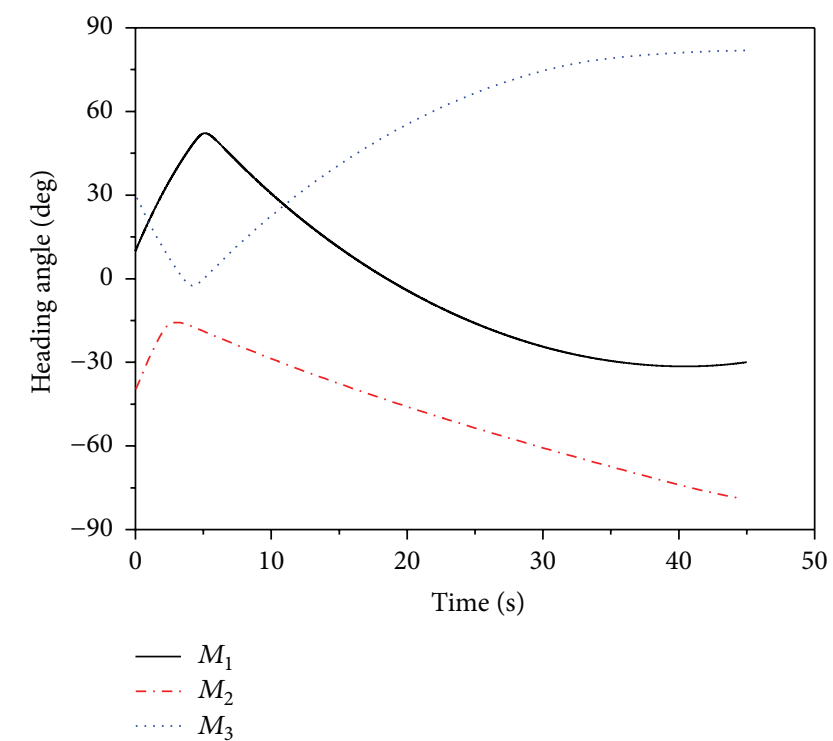

(c) Heading angle

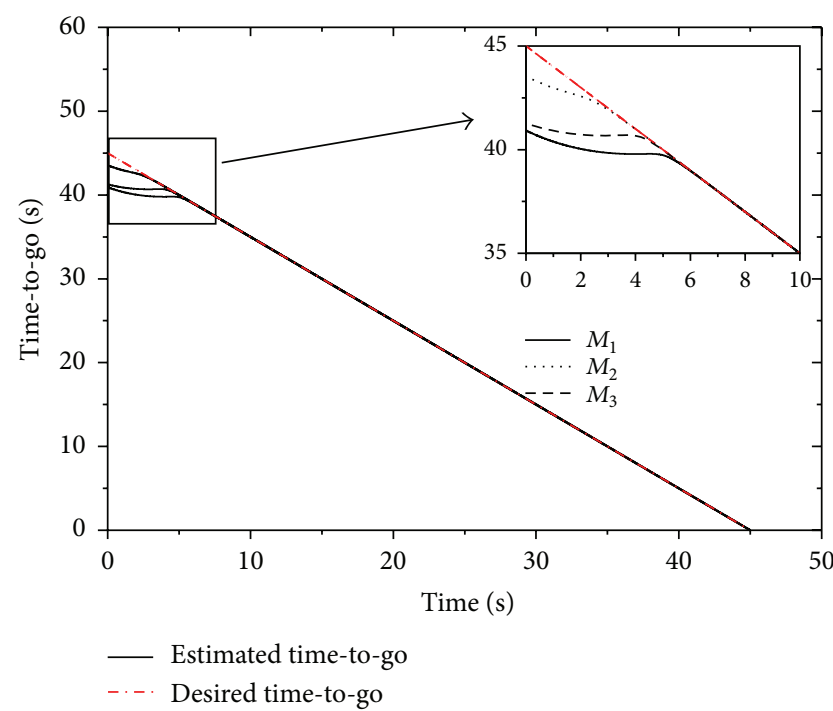

(b) Time-to-go

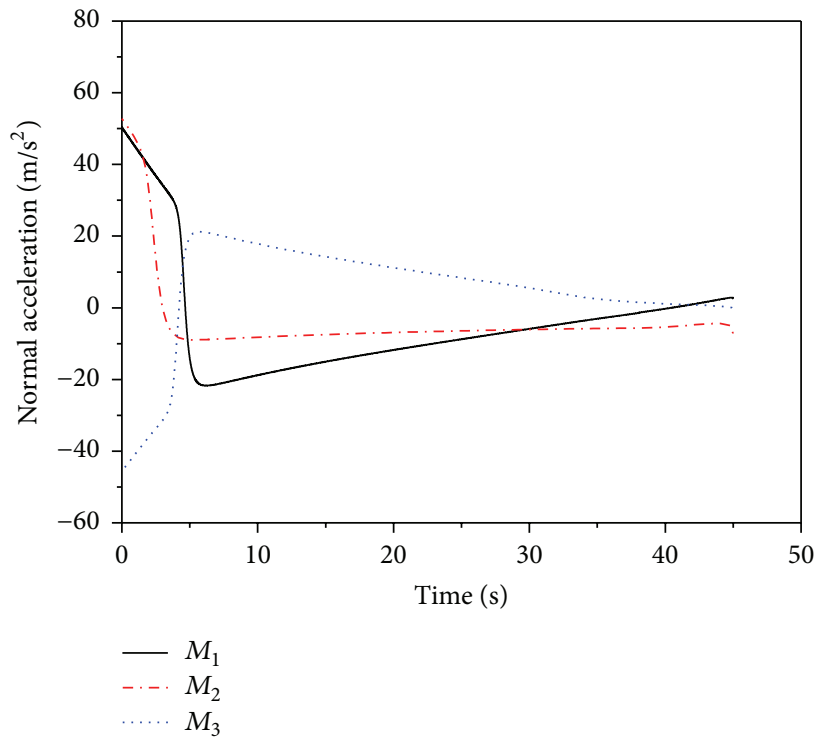

(d) Missile acceleration

FIGURE 6: Simulations results considering a salvo attack against a stationary target.

TABLE 2: Simulation parameters for salvo attack.

\begin{tabular}{lccc}
\hline & Missile 1 & Missile 2 & Missile 3 \\
\hline Position $X / \mathrm{m}$ & 0 & 3000 & 4000 \\
Position $Y / \mathrm{m}$ & 500 & 8000 & -8000 \\
Heading angle/deg & 10 & -40 & 30 \\
Desired impact angle/deg & -30 & -80 & 80 \\
Desired impact time/s & 45 & 45 & 45 \\
\hline
\end{tabular}

parameters are given in Table 2. Figure 6(a) illustrates the salvo attack trajectories used by ITACG compared with PNG. It can be seen that the trajectories used by ITACG have a greater curvature than those with PNG. Figure 6(b) shows that the proposed guidance law reduces the impact time dispersion with negligible error. The impact angles assigned to each missile are all achieved at the final time, as shown in Figure 6(c). From Figure 6(d), missiles need larger accelerations at the beginning to enable the system to move to the sliding mode.

4.2. With Autopilot Dynamics. In this subsection, first-order uncompensated autopilot dynamics are considered with a time constant $\tau_{m}$. The autopilot dynamics lag can be expressed as

$$
a_{m}=\frac{1}{\tau_{m} s+1} a_{c},
$$

where $a_{c}$ and $a_{m}$ are the acceleration command and the lateral acceleration, respectively. 


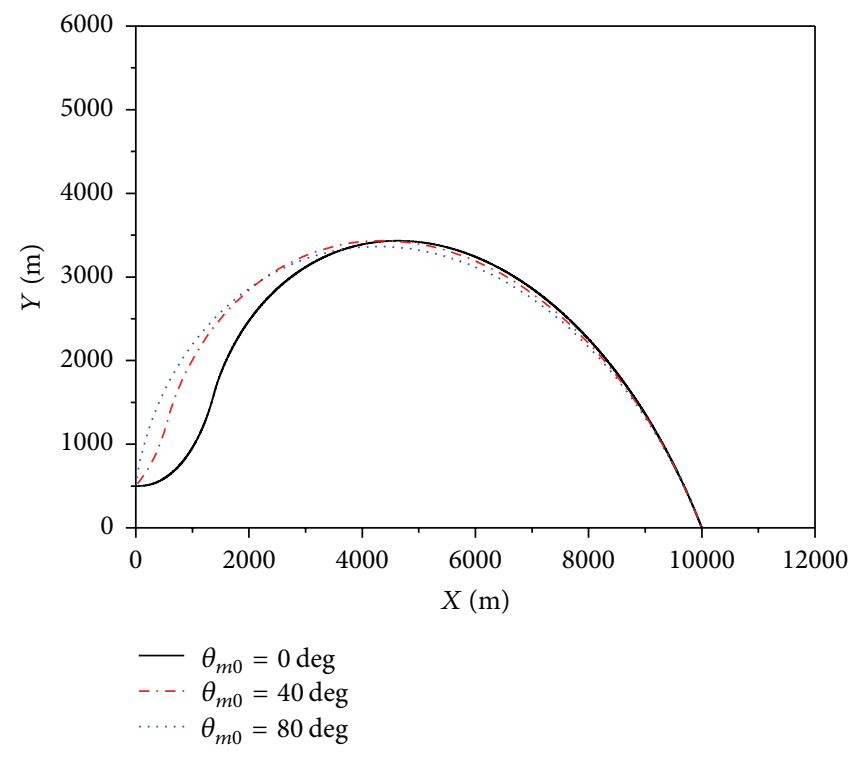

(a) Missile trajectory

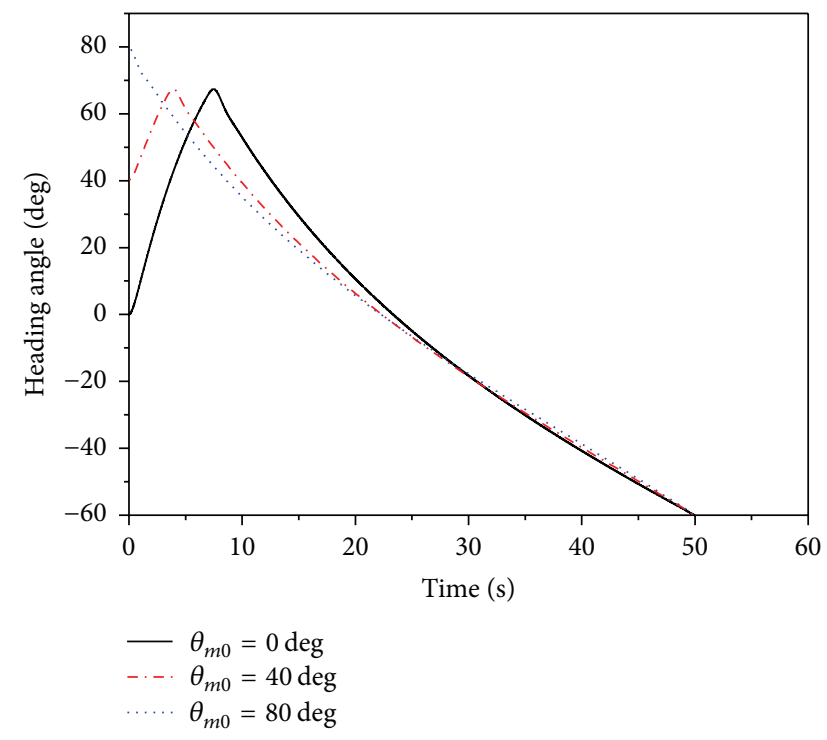

(c) Heading angle
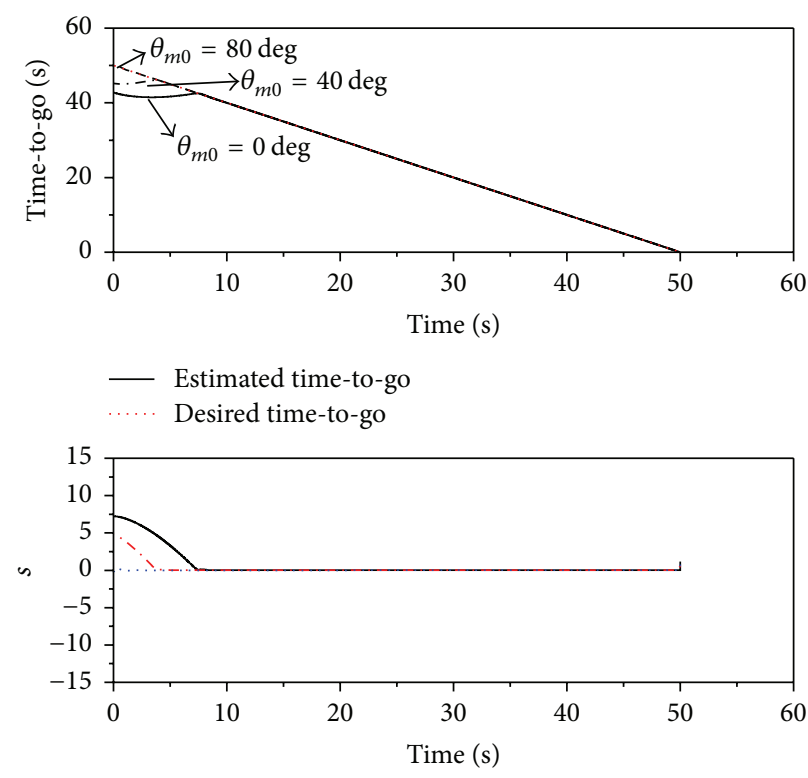

$$
\begin{aligned}
-\theta_{m 0} & =0 \mathrm{deg} \\
\cdots-\theta_{m 0} & =40 \mathrm{deg} \\
\cdots \cdots \theta_{m 0} & =80 \mathrm{deg}
\end{aligned}
$$

(b) Time-to-go and occurrence of sliding mode

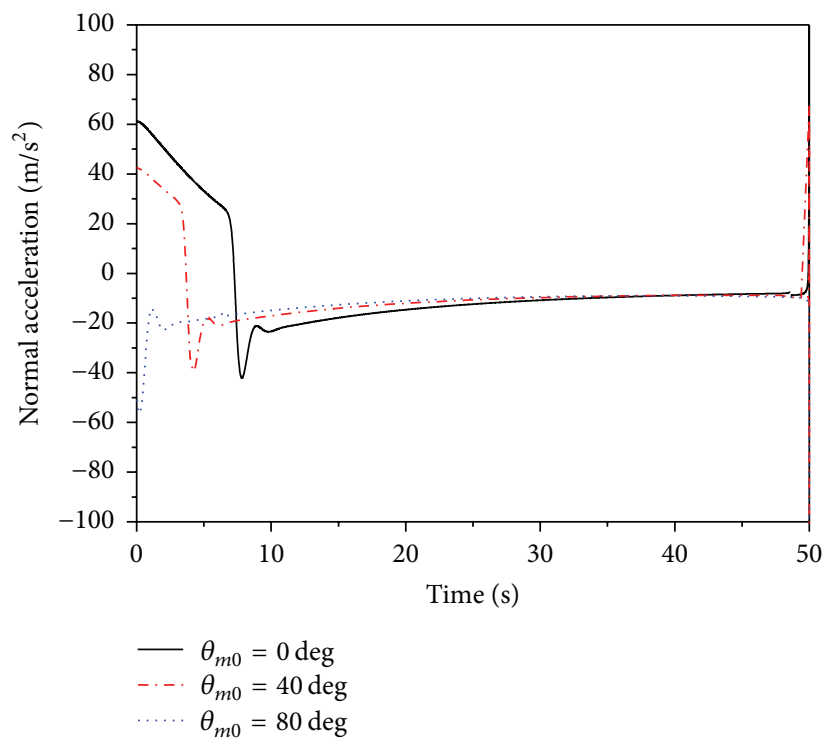

(d) Missile acceleration

FIGURE 7: Simulation results considering various launch angles with autopilot dynamics.

Note that, in the presence of autopilot lag, the coefficients in (13) are no longer constant. They should be updated as time-varying parameters, which are given as

$$
\begin{aligned}
& a(x)=\frac{x_{T}^{3}}{\left(x_{T}-x\right)^{2}} \lambda^{\prime}-\frac{2 x_{T}^{3}\left(\theta_{d}-\lambda\right)}{\left(x_{T}-x\right)^{3}}, \\
& b(x)=\frac{3 x_{T}^{2}\left(x+x_{T}\right)\left(\theta_{d}-\lambda\right)}{\left(x_{T}-x\right)^{3}}-\frac{x_{T}^{2}\left(x+2 x_{T}\right)}{\left(x_{T}-x\right)^{2}} \lambda^{\prime},
\end{aligned}
$$




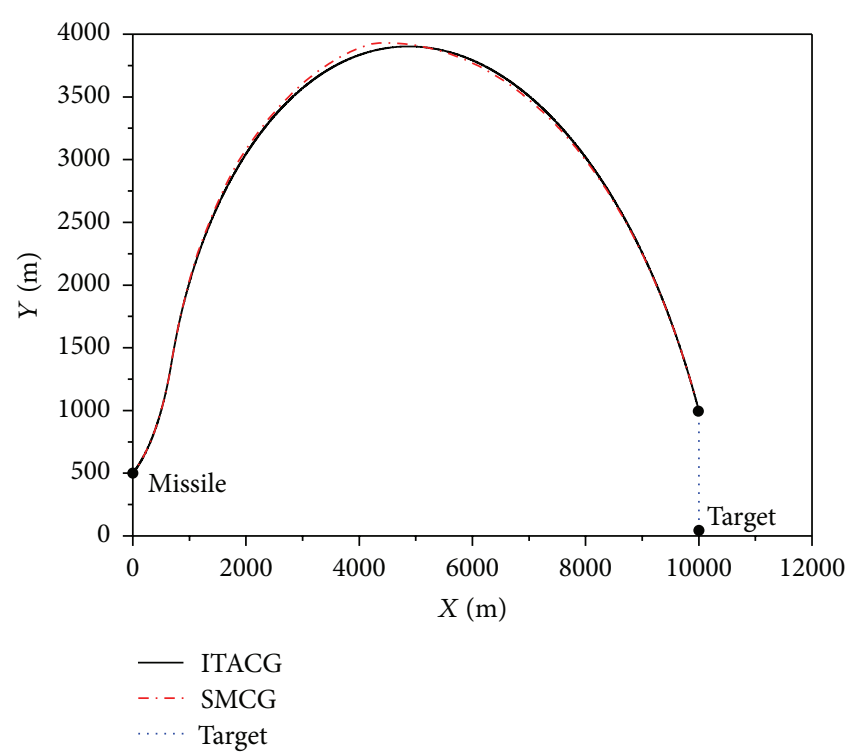

(a) Missile trajectory

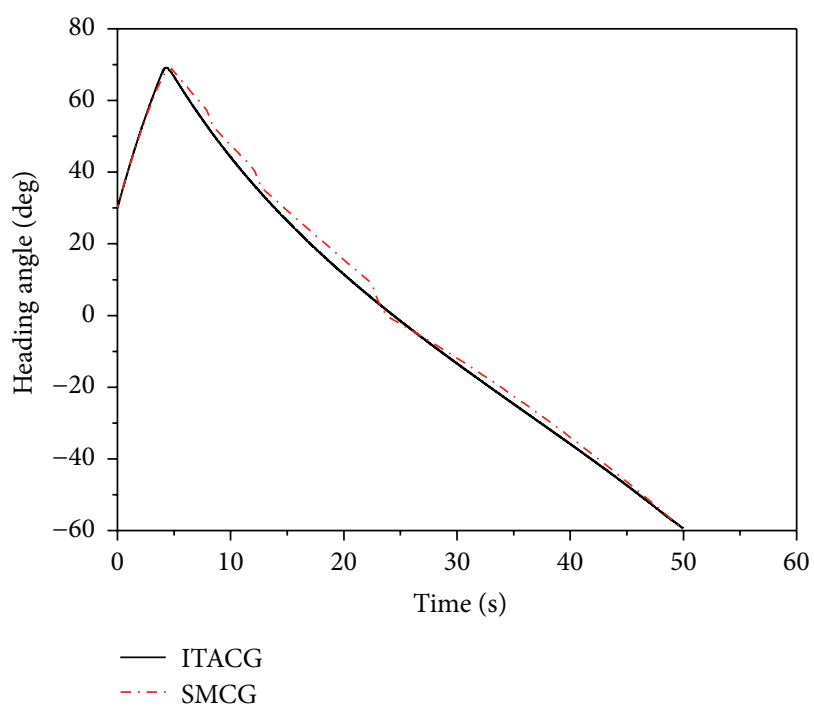

(c) Heading angle
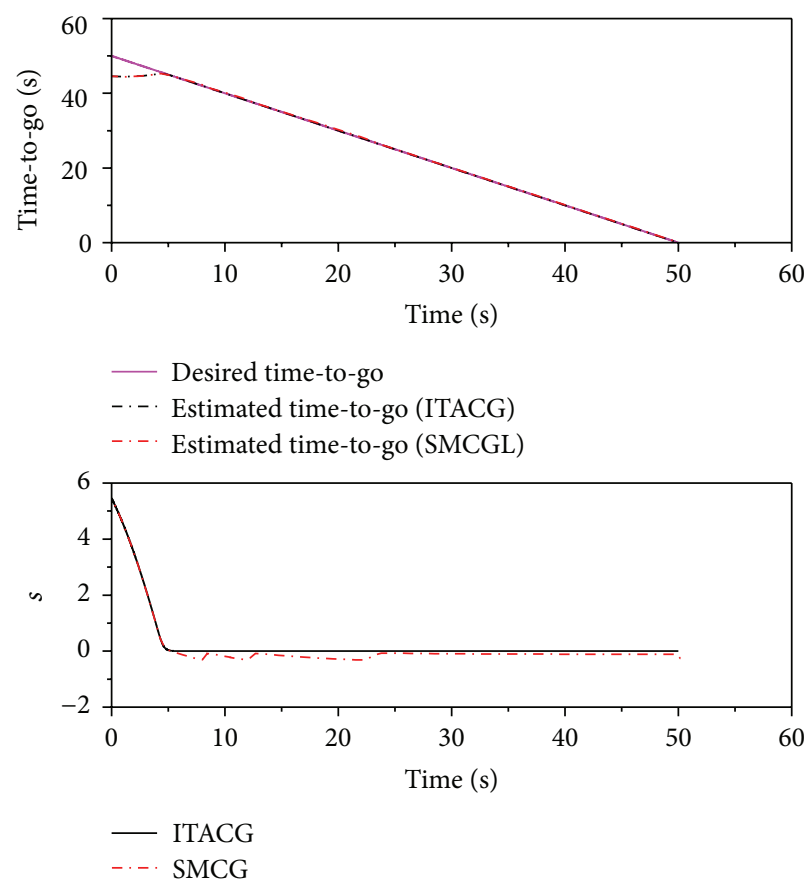

(b) Time-to-go and occurrence of sliding mode

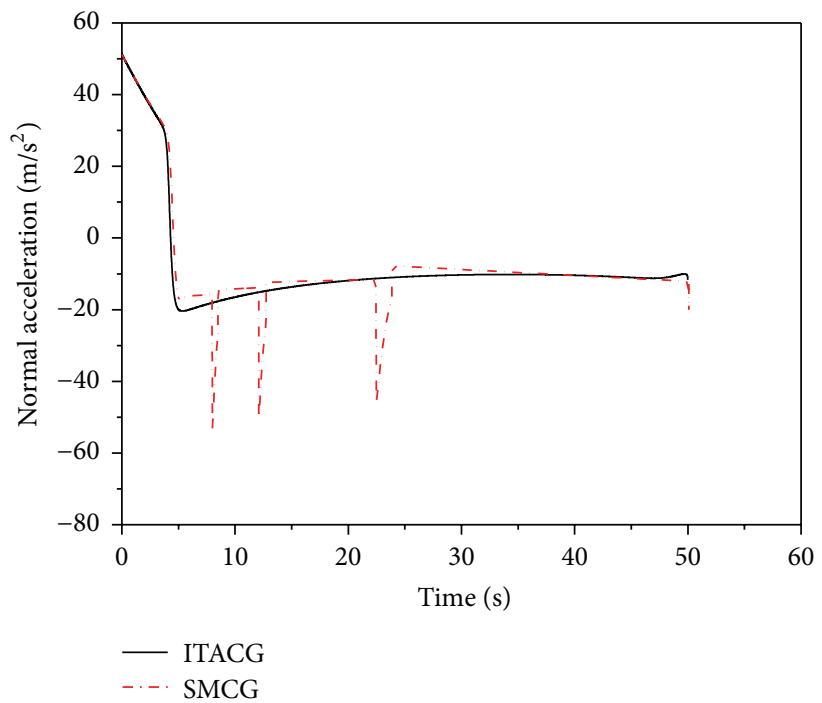

(d) Missile acceleration

FIGURE 8: Simulation results for a nonmaneuvering target.

Simulations are carried out for different initial heading angles with autopilot time constant $\tau_{m}=0.3 \mathrm{~s}$. With the same initial position and other constraints as in Table 1, different launch angles, 0, 40 and $80 \mathrm{deg}$, are considered. Figure 7 shows that the missile successfully intercepts the target at the desired impact time and angle with different launch angles. The impact time error is less than $1 \times 10^{-2} \mathrm{~s}$ for all the cases. And the impact angle error is less than $0.1 \mathrm{deg}$. This simulation highlights the robustness of the proposed guidance law with respect to uncompensated autopilot delays.
4.3. Nonmaneuvering Target. This subsection demonstrates the effectiveness of the proposed ITACG against a nonmaneuvering target. The target is assumed to move with a velocity of $20 \mathrm{~m} / \mathrm{s}$, which is a typical cruise speed for a modern warfare ship. The heading angle of the target is $90 \mathrm{deg}$, and the initial conditions of the missile and target are given in Table 3 . Figure 8 indicates that the proposed ITACG successfully achieves the desired impact time and impact angle when used against a moving target. The impact time error of ITACG is $0.01 \mathrm{~s}$, which is smaller than the $0.29 \mathrm{~s}$ error 
TABLE 3: Simulation parameters against a moving target.

\begin{tabular}{lcccccc}
\hline$\left(x_{0}, y_{0}\right) / \mathrm{m}$ & $\theta_{0} / \mathrm{deg}$ & $\theta_{f} / \mathrm{deg}$ & $T_{d} / \mathrm{s}$ & $\left(x_{T}, y_{T}\right) / \mathrm{m}$ & $V_{T} /(\mathrm{m} / \mathrm{s})$ & $\theta_{T} / \mathrm{deg}$ \\
\hline$(0,500)$ & 30 & -60 & 50 & $(10000,0)$ & 20 & 90 \\
\hline
\end{tabular}

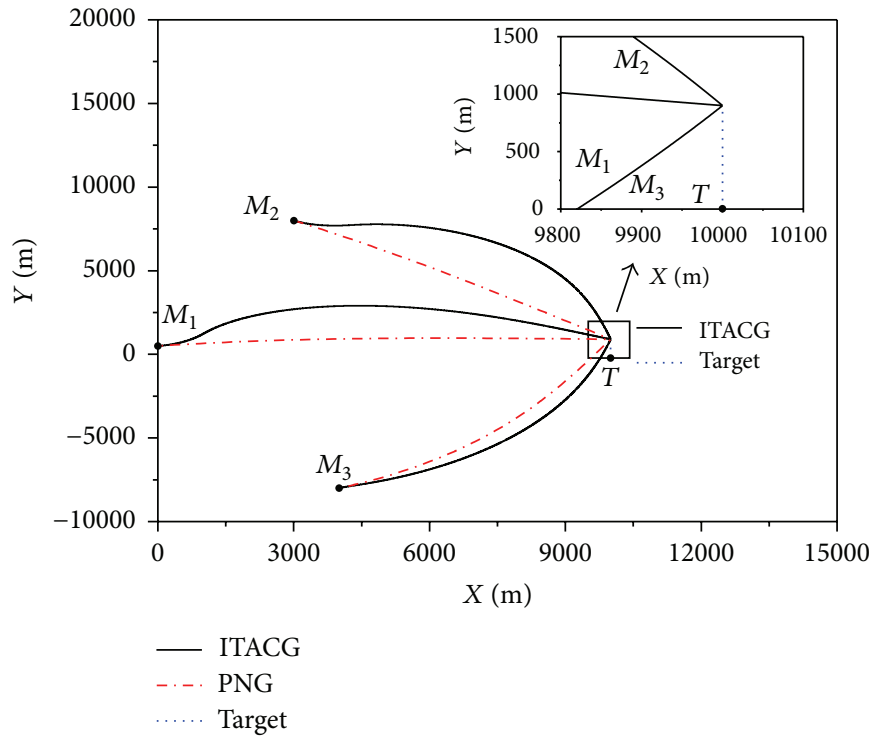

(a) Missile trajectory

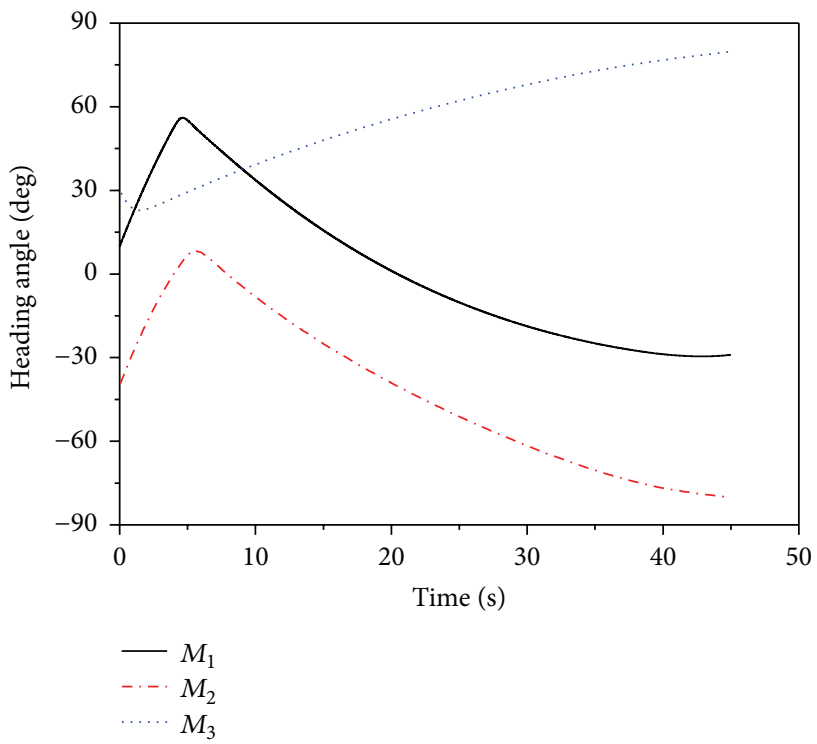

(c) Heading angle

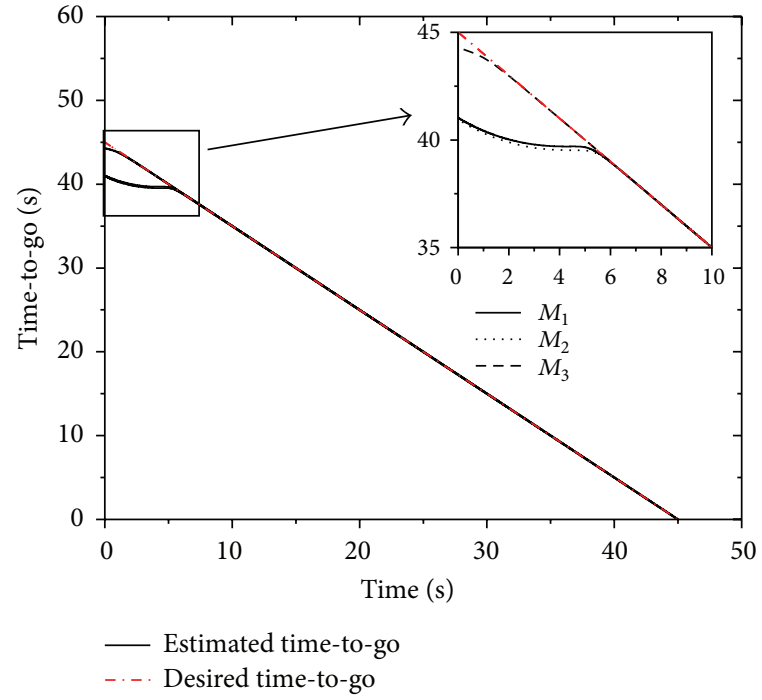

(b) Time-to-go

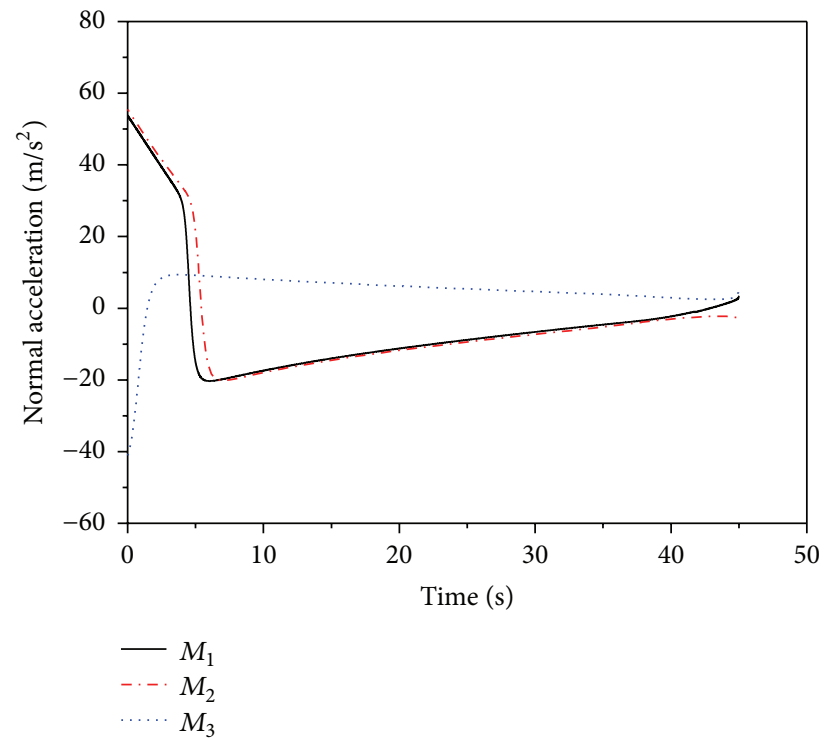

(d) Missile acceleration

FIGURE 9: Simulation results considering a salvo attack against a nonmaneuvering target.

obtained using SMCG. Figure 8(d) shows that the proposed guidance law avoids the abrupt jumps of lateral acceleration.

A further simulation is performed in a salvo attack scenario. As before, the target is moving with a velocity of $20 \mathrm{~m} / \mathrm{s}$ and a heading angle of $90 \mathrm{deg}$. The other simulation conditions are the same as in the previous salvo attack scenario against a stationary target. Figure 9 shows that each missile successfully intercepts the moving target at the desired time with the correct impact angle.

\section{Conclusion}

In this paper, a novel guidance law based on sliding mode control is presented to achieve both impact time and angle control. The sliding mode surface is formed using the LOS rate error and the time-to-go error. The LOS rate shaping method is designed to realize the terminal angle requirement and the time-to-go derivative estimation is used to minimize the impact time error. Simulations of various engagement 
scenarios demonstrate that the proposed guidance law successfully controls the impact time and angle against both stationary and nonmaneuvering targets. Further simulations show that the proposed guidance law also provides excellent performance considering autopilot dynamics. Future studies will consider additional constraints with regard to this guidance law, such as the seeker's field-of-view limit.

\section{Competing Interests}

The authors declare that there are no competing interests regarding the publication of this article.

\section{References}

[1] Y. A. Zhang, X. L. Wang, and H. L. Wu, "Impact time control guidance law with field of view constraint," Aerospace Science and Technology, vol. 39, pp. 361-369, 2014.

[2] T.-H. Kim, C.-H. Lee, I.-S. Jeon, and M.-J. Tahk, "Augmented polynomial guidance with impact time and angle constraints," IEEE Transactions on Aerospace and Electronic Systems, vol. 49, no. 4, pp. 2806-2817, 2013.

[3] S. Kang and H. J. Kim, "Differential game missile guidance with impact angle and time constraints," IFAC Proceedings Volumes, vol. 44, no. 1, pp. 3920-3925, 2011.

[4] M. Kim and K. V. Grider, "Terminal guidance for impact attitude angle constrained flight trajectories," IEEE Transactions on Aerospace and Electronic Systems, vol. 9, no. 6, pp. 852-859, 1973.

[5] B. S. Kim, J. G. Lee, and H. S. Han, "Biased PNG law for impact with angular constraint," IEEE Transactions on Aerospace and Electronic Systems, vol. 34, no. 1, pp. 277-288, 1998.

[6] C.-K. Ryoo, H. Cho, and M.-J. Tahk, "Optimal guidance laws with terminal impact angle constraint," Journal of Guidance, Control, and Dynamics, vol. 28, no. 4, pp. 724-732, 2005.

[7] S. R. Kumar, S. Rao, and D. Ghose, "Nonsingular terminal sliding mode guidance with impact angle constraints," Journal of Guidance, Control, and Dynamics, vol. 37, no. 4, pp. 1114-1130, 2014.

[8] S. He, D. Lin, and J. Wang, "Continuous second-order sliding mode based impact angle guidance law," Aerospace Science and Technology, vol. 41, pp. 199-208, 2015.

[9] Y. Zhang, M. Sun, and Z. Chen, "Finite-time convergent guidance law with impact angle constraint based on slidingmode control," Nonlinear Dynamics, vol. 70, no. 1, pp. 619-625, 2012.

[10] I.-S. Jeon, J.-I. Lee, and M.-J. Tahk, "Impact-time-control guidance law for anti-ship missiles," IEEE Transactions on Control Systems Technology, vol. 14, no. 2, pp. 260-266, 2006.

[11] S. Zhao and R. Zhou, "Cooperative guidance for multimissile salvo attack," Chinese Journal of Aeronautics, vol. 21, no. 6, pp. 533-539, 2008.

[12] S. R. Kumar and D. Ghose, "Impact time guidance for large heading errors using sliding mode control," IEEE Transactions on Aerospace and Electronic Systems, vol. 51, no. 4, pp. 3123-3138, 2015.

[13] D. Cho, H. J. Kim, and M.-J. Tahk, "Nonsingular sliding mode guidance for impact time control," Journal of Guidance, Control, and Dynamics, vol. 39, no. 1, pp. 61-68, 2016.
[14] X. Wei, Y. Wang, S. Dong, and L. Liu, "A three-dimensional cooperative guidance law of multimissile system," International Journal of Aerospace Engineering, vol. 2015, Article ID 479427, 8 pages, 2015.

[15] J. Zhao, R. Zhou, and Z. Dong, "Three-dimensional cooperative guidance laws against stationary and maneuvering targets," Chinese Journal of Aeronautics, vol. 28, no. 4, pp. 1104-1120, 2015.

[16] J. Bokyung and K. Youdan, "Guidance laws for anti-ship missiles using impact angle and impact time," in Proceedings of the AIAA Guidance, Navigation, and Control Conference and Exhibit, American Institute of Aeronautics and Astronautics, Keystone, Colo, USA, August 2006.

[17] Y. A. Zhang, G. X. Ma, and A. L. Liu, "Guidance law with impact time and impact angle constraints," Chinese Journal of Aeronautics, vol. 26, no. 4, pp. 960-966, 2013.

[18] N. Harl and S. N. Balakrishnan, "Impact time and angle guidance with sliding mode control," IEEE Transactions on Control Systems Technology, vol. 20, no. 6, pp. 1436-1449, 2012.

[19] S. R. Kumar and D. Ghose, "Impact time and angle control guidance," in Proceedings of the AIAA Guidance, Navigation, and Control Conference, AIAA, Kissimmee, Fla, USA, January 2015.

[20] C.-H. Lee, T.-H. Kim, and M.-J. Tahk, "Interception angle control guidance using proportional navigation with error feedback," Journal of Guidance, Control, and Dynamics, vol. 36, no. 5, pp. 1556-1561, 2013.

[21] I.-S. Jeon, J.-I. Lee, and M.-J. Tahk, "Homing guidance law for cooperative attack of multiple missiles," Journal of Guidance, Control, and Dynamics, vol. 33, no. 1, pp. 275-280, 2010.

[22] S. R. Kumar and D. Ghose, "Sliding mode control based guidance law with impact time constraints," in Proceedings of the 1st American Control Conference (ACC '13), pp. 5760-5765, June 2013. 


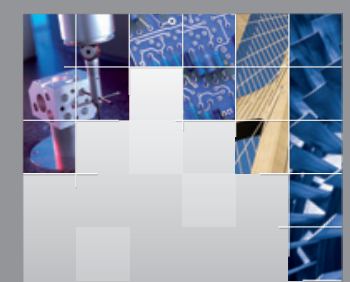

\section{Enfincering}
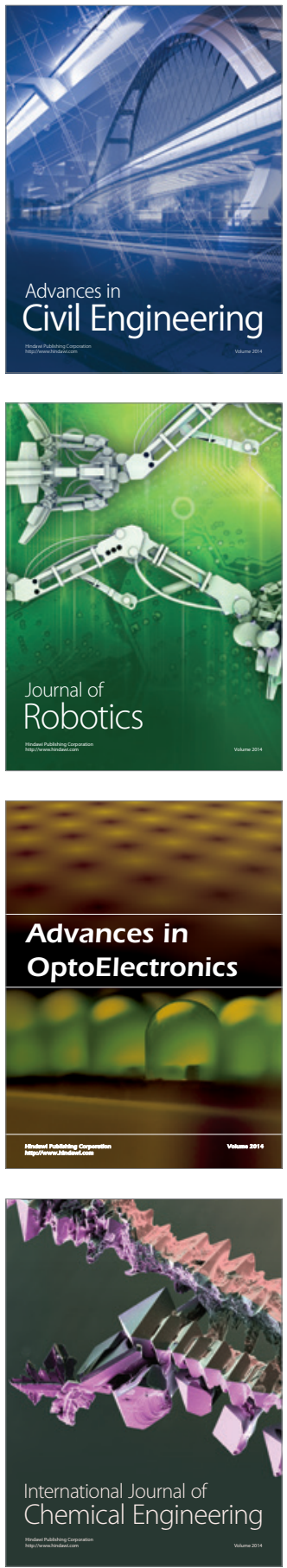

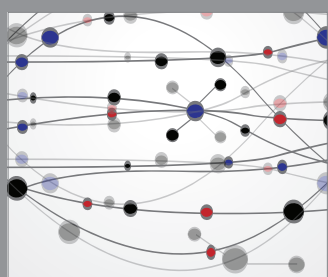

The Scientific World Journal

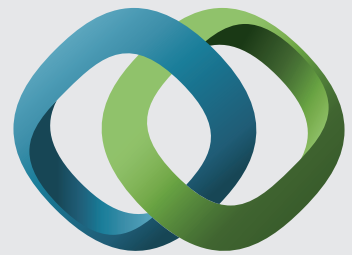

\section{Hindawi}

Submit your manuscripts at

http://www.hindawi.com
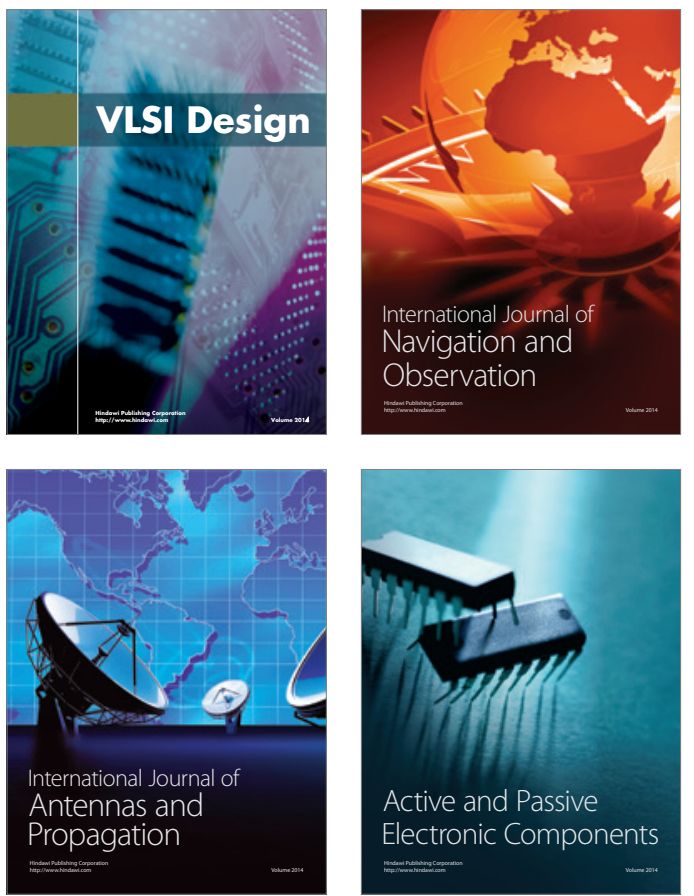
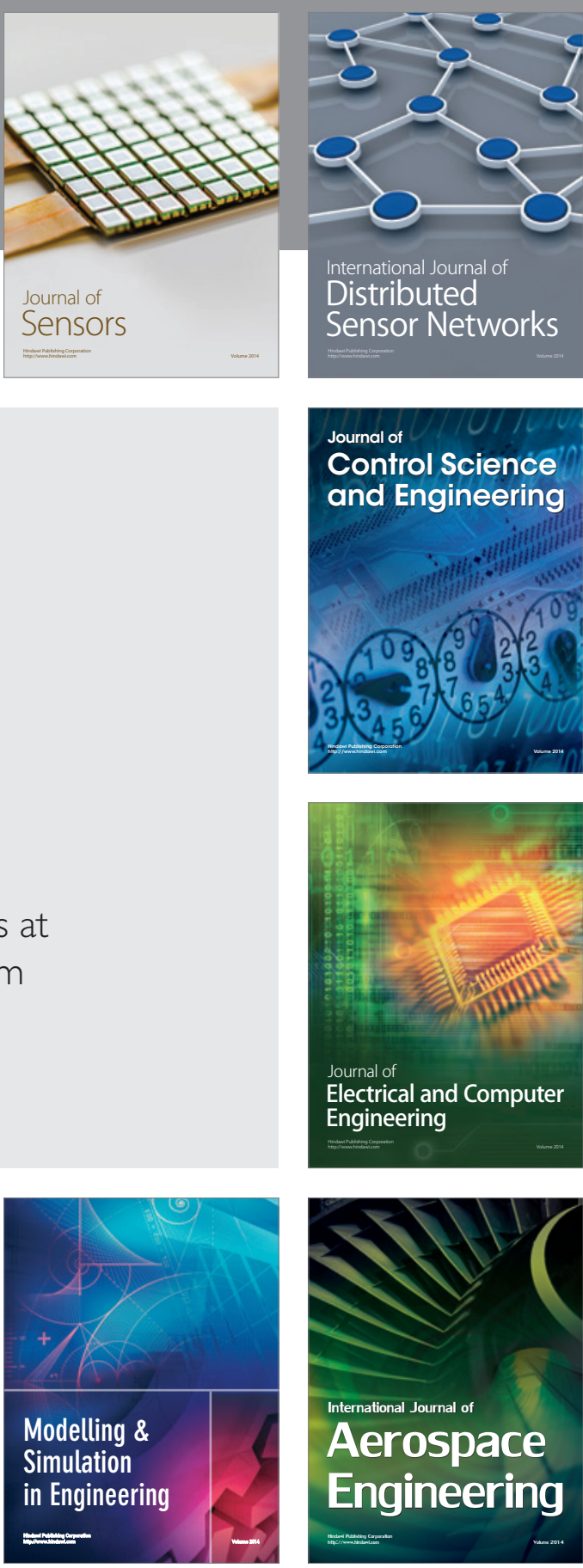

International Journal of

Distributed

Sensor Networks

Journal of

Control Science

and Engineering
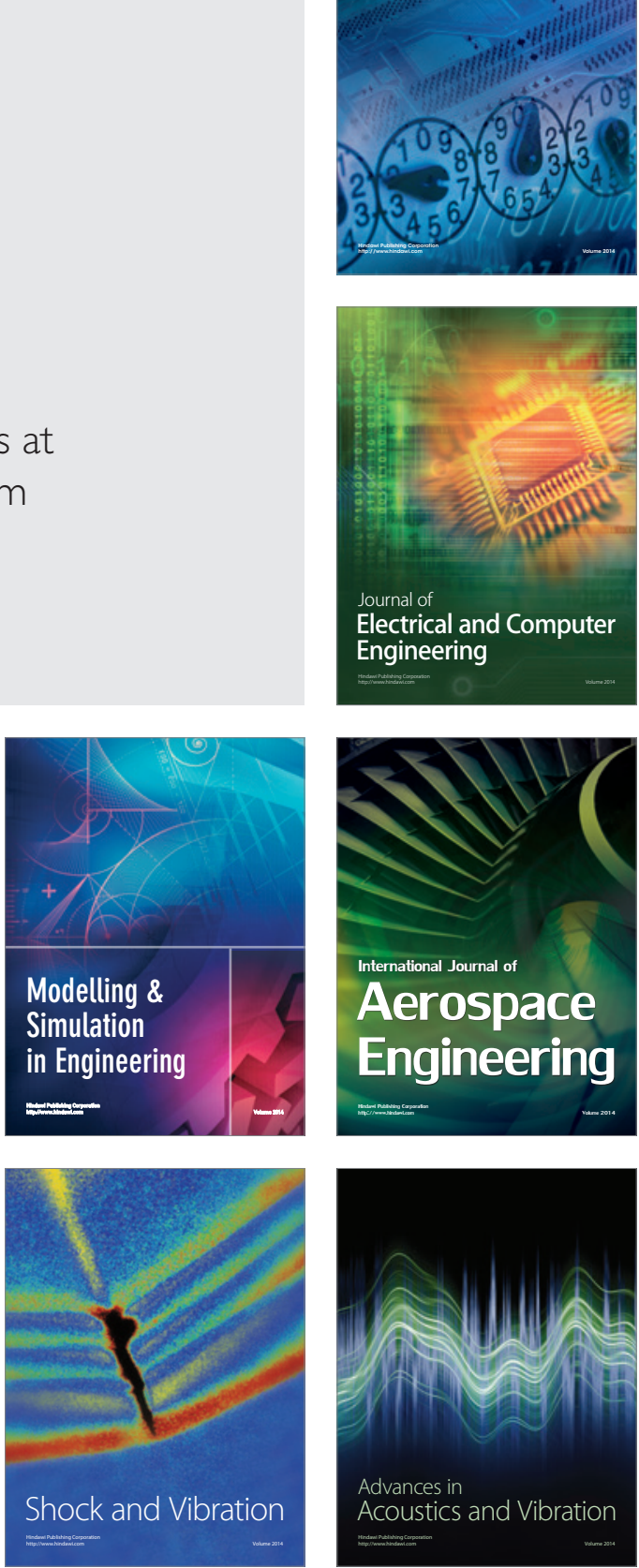